\title{
Postnikov extensions of ring spectra
}

\author{
DANIEL DUGGer \\ BROOKE SHIPLEY
}

\begin{abstract}
We give a functorial construction of $k$-invariants for ring spectra and use these to classify extensions in the Postnikov tower of a ring spectrum.
\end{abstract}

55P43; 55S45

\section{Introduction}

This paper concerns $k$-invariants for ring spectra and their role in classifying Postnikov extensions. Recall that a connective ring spectrum $R$ has a Postnikov tower

$$
\cdots \rightarrow P_{2} R \rightarrow P_{1} R \rightarrow P_{0} R \rightarrow *
$$

in the homotopy category of ring spectra. The levels come equipped with compatible maps $R \rightarrow P_{n} R$, and the $n$-th level is characterized by having $\pi_{i}\left(P_{n} R\right)=0$ for $i>n$, together with the fact that $\pi_{i}(R) \rightarrow \pi_{i}\left(P_{n} R\right)$ is an isomorphism for $i \leq n$. In this paper we produce $k$-invariants for the levels of this tower and explain their role in the following problem: if one only knows $P_{n-1} R$ together with $\pi_{n}(R)$ as a $\pi_{0}(R)$-bimodule, what are the possibilities for $P_{n} R$ ? Corollary 1.4 shows in what sense the possibilities are classified by $k$-invariants.

\subsection{Classical $k$-invariants}

To explain our results further, it's useful to briefly recall the situation for ordinary topological spaces. If $X$ is a space, let $P_{n} X$ be the $n$-th Postnikov section of $X$. The $k$-invariant is a map $P_{n-1} X \rightarrow K\left(\pi_{n} X, n+1\right)$ and the homotopy fiber of this map is weakly equivalent to $P_{n} X$. So $P_{n} X$ can be recovered from the $k$-invariant, and in fact the $k$-invariant only depends on $P_{n} X$. One is tempted to say that the possibilities for $P_{n} X$ are classified by the possible $k$-invariants, but this is where some care is needed.

To clarify the situation, it's useful to set $C=P_{n-1} X$ and $M=\pi_{n} X$. By a Postnikov $n$-extension of $C$ (of type $M$ ) we mean a space $Y$ together with a map $Y \rightarrow C$ such that $\pi_{i}(Y) \rightarrow \pi_{i}(C)$ is an isomorphism for $i \leq n-1, \pi_{n} Y \cong M$, and $\pi_{i} Y=0$ for 
$i>n$. Note that the isomorphism $\pi_{n} Y \cong M$ is not part of the data. The Postnikov $n$-extensions form a category, in which a map from $Y \rightarrow C$ to $Y^{\prime} \rightarrow C$ is a weak equivalence $Y \rightarrow Y^{\prime}$ making the evident triangle commute. We'll denote this category $\mathcal{M}(C, M, n)$.

For convenience, suppose $C$ is simply connected (so we don't have to worry about the $\pi_{1} C$ actions). One is tempted to claim that the connected components of $\mathcal{M}(C, M, n)$ are in bijective correspondence with the set of homotopy classes [C, $K(M, n+1)]$. Unfortunately, this isn't quite the case. Note that the group Aut $M$ of abelian group automorphisms acts on $K(M, n+1)$ and hence on $[C, K(M, n+1)]$. If a certain $k$-invariant $C \rightarrow K(M, n+1)$ is "twisted" by an automorphism of $M$, it gives rise to a weakly equivalent extension of $C$. The correct statement, it then turns out, is that if $C$ is simply connected there is a bijection

$$
\pi_{0} \mathcal{M}(C, M, n) \cong[C, K(M, n+1)] / \operatorname{Aut}(M)
$$

This statement is best proven by upgrading it to a statement about the homotopy type of $\mathcal{M}(C, M, n)$ (where by the homotopy type of a category we always mean the homotopy type of its nerve). One can prove that there is a homotopy fiber sequence

$$
\operatorname{Map}(C, K(M, n+1)) \rightarrow \mathcal{M}(C, M, n) \rightarrow B \operatorname{Aut}(M),
$$

and the resulting long exact sequence of homotopy groups gives the identification of $\pi_{0} \mathcal{M}(C, M, n)$ cited above.

For a proof of this homotopy fiber sequence (together with a version when $C$ is not simply connected) we refer the reader to Blanc, Dwyer and Goerss [4, Sections 2,3]. An important part of the proof is having a simple, functorial construction of the $k-$ invariant, and we now describe this. If $p: Y \rightarrow C$ is a Postnikov $n$-extension of type $M$, let $D$ denote the homotopy cofiber of $p$. One can prove by a Blakers-Massey type result that $\pi_{i}(D)=0$ for $i \leq n$, whereas $\pi_{n+1} D \cong \pi_{n} Y \cong M$. Then $P_{n+1} D$ is an Eilenberg-Mac Lane space $K(M, n+1)$, and our $k$-invariant for $Y$ is the composite

$$
C \rightarrow D \rightarrow P_{n+1} D
$$

Note that in some sense this is not really a $k$-invariant, as one does not have a specified weak equivalence $P_{n+1} D \simeq K(M, n+1)$. One can prove that different weak equivalences differ by an element of $\operatorname{Aut}(M)$, and this shows that one has a well-defined element of the orbit space $[C, K(M, n+1)] / \operatorname{Aut}(M)$. 


\subsection{Results for ring spectra}

Now we jump into the category of ring spectra, and state our main results. In this paper we always work in the category of symmetric spectra from [13]. So "ring spectrum" means "symmetric ring spectrum".

Fix $n \geq 1$. Let $C$ be a connective ring spectrum such that $P_{n-1} C \simeq C$, and let $M$ be a $\pi_{0} C$-bimodule. By a Postnikov extension of $C$ of type $(M, n)$ one means a ring map $Y \rightarrow C$ such that

(i) $\pi_{i} Y=0$ for $i>n$,

(ii) $\pi_{i} Y \rightarrow \pi_{i} C$ is an isomorphism for $i \leq n-1$,

(iii) $\pi_{n} Y \cong M$ as $\pi_{0} Y$-bimodules (where $M$ becomes a $\pi_{0} Y$-bimodule via the isomorphism $\pi_{0} Y \rightarrow \pi_{0} C$ ).

A map of Postnikov extensions from $Y \rightarrow C$ to $Y^{\prime} \rightarrow C$ is a weak equivalence of ring spectra $Y \rightarrow Y^{\prime}$ making the triangle commute. Denote the resulting category by $\mathcal{M}(C+(M, n))$. We call this the moduli space (or moduli category) of Postnikov extensions of $C$ of type $(M, n)$. Note that we are not assuming that $C$ is fibrant here.

We next identify the analogues of Eilenberg-Mac Lane spaces. Given a $C$-bimodule $W$, one can construct a ring spectrum $C \vee W$ whose underlying spectrum is the wedge and where the multiplication comes from the bimodule structure on $W-$ so $W$ squares to zero under this product. We call $C \vee W$ the trivial square zero extension of $C$ by $W$.

Given our $\pi_{0}(C)$-bimodule $M$, there is a $C$-bimodule $H M$ for which $\pi_{0}(H M) \cong$ $M$ and $\pi_{i}(H M)=0$ for $i \neq 0$. In fact, all such bimodules are weakly equivalent (in the category of $C$-bimodules). One gets resulting bimodules $\Sigma^{i}(H M)$ for all $i$, and therefore ring spectra $C \vee \Sigma^{i}(H M)$. Throughout the paper we will abuse notation and simplify $H M$ to just $M$-thus, we will write $C \vee \Sigma^{i} M$ for $C \vee \Sigma^{i} H M$.

Here is our main theorem:

1.3 Theorem Given $C$ and $M$ as above, there is a homotopy fiber sequence

$$
\underline{\operatorname{Ring}_{/ C}}\left(C, C \vee \Sigma^{n+1} M\right) \rightarrow \mathcal{M}(C+(M, n)) \rightarrow B \operatorname{Aut}(M)
$$

where $\operatorname{Ring}_{/ C}(X, Y)$ denotes the homotopy mapping space from $X$ to $Y$ in the category of ring spectra over $C$ and $\operatorname{Aut}(M)$ is the group of $\pi_{0}(C)$-bimodule automorphisms of $M$. 


\subsection{Corollary There is a bijection of sets}

$$
\pi_{0} \mathcal{M}(C+(M, n)) \cong\left[\operatorname{Ho}(\mathcal{R i n g} / C)\left(C, C \vee \Sigma^{n+1} M\right)\right] / \operatorname{Aut}(M) .
$$

In the context of these results, the main difference between ring spectra and ordinary topological spaces is that there are no absolute cohomology theories for ring spectra. When dealing with ring spectra, one always deals with relative cohomology theories. (For a nice explanation of this phenomenon in the commutative case, see the introduction to Basterra and Mandell [2].) Thus, in the above results one is forced to always work over $C$ : the analogue of the Eilenberg-Mac Lane space is the ring spectrum $C \vee \Sigma^{n+1} M$, and the mapping spaces must be computed in the category of ring spectra over $C$. Aside from these differences, the statements for ring spectra and topological spaces are quite similar.

The set of homotopy classes appearing in Corollary 1.4 can be identified with a topological Hochschild cohomology group. In this way one sees that $\mathrm{THH}^{*}$ is the natural receptor for $k$-invariants of associative ring spectra. This perspective also simplifies calculations, since topological Hochschild cohomology involves a homotopy category of bimodules instead of a homotopy category of rings. All of this is discussed in Section 8.5, and we have the following restatement of Corollary 1.4.

1.5 Proposition Let $C$ and $M$ be as above. Assuming that $C$ is cofibrant as an underlying spectrum, one has a bijection

$$
\pi_{0} \mathcal{M}(C+(M, n)) \cong \mathrm{THH}^{n+2}(C, M) / \operatorname{Aut}(M) .
$$

Finally, we remark that the above results can actually be extended, so that they apply not just to ring spectra but to algebras over a given connective, commutative ring spectrum $R$. This is the form in which we will actually prove them (see Theorem 8.1 and Proposition 8.8). Moreover, all the results of the paper apply equally well to the category of differential graded algebras over a commutative ground ring $k$. Our proofs all adapt essentially verbatim, or else one can use that the homotopy theory of dgas over $k$ is equivalent to that of algebras over the Eilenberg-Mac Lane ring spectrum $H k$ (this is proven by the second author in [19]).

1.6 Remark Both Theorem 1.3 and Corollary 1.4 can also be rewritten in terms of ring spectra over $P_{0} C$ (the zero-th Postnikov stage of $C$ ) rather than ring spectra over $C$. For this, see Proposition 8.6. 


\subsection{Some background}

Corollary 1.4 was needed in our paper [7], and we at first believed this result to be obvious. Our attempts to give a careful proof, however, always seemed to fail. A construction of $k$-invariants for ring spectra had already been given in Lazarev [14], but that construction does not seems well-suited for the above classification questions. A construction of $k$-invariants for commutative ring spectra appeared in Basterra [1]; while this construction also did not meet all of our needs, many of the techniques of [1] are used in our Section 6.

Eventually we discovered Blanc, Dwyer and Goerss [4], which applied the Dwyer-Kan moduli space technology to the classification of Postnikov extensions in a related context. It will be clear to the reader that the basic methods in the present paper are heavily influenced by [4] (there is one main difference, discussed in Remark 8.4). However, in order to carry out the program of [4] we have had to straighten out many points about ring spectra along the way. One of the main things the reader will find here is a careful, functorial construction of $k$-invariants for ring spectra. We also provide a careful proof of a Blakers-Massey theorem for ring spectra in Appendix A. We thank a helpful referee and Mike Mandell for suggestions which improved our presentation of this result.

\subsection{Notation and terminology}

If $\mathcal{C}$ is a category then we write $\mathcal{C}(X, Y)$ for $\operatorname{Hom}_{\mathcal{C}}(X, Y)$. If $\mathcal{C}$ is a simplicial model category we write $\operatorname{Map}_{\mathcal{C}}(X, Y)$ for the simplicial function complex. If $\mathcal{C}$ is a model category then $\underline{\mathrm{e}}(X, Y)$ denotes a homotopy function complex from $X$ to $Y$. The phrase "homotopy function complex" indicates a construction which has the correct homotopy type even if $X$ is not cofibrant and $Y$ is not fibrant. To fix a particular construction, we use the hammock localization of Dwyer and Kan [9].

If $R$ is a commutative ring spectrum then there are model category structures on $R$-Mod and $R-\mathcal{A l g}$ provided by Schwede and the second author [18]; in each case the fibrations and weak equivalences are determined by the forgetful functor to symmetric spectra. We use these model categories throughout the paper.

To every category $\mathcal{C}$ one can associate a simplicial set $|\mathcal{C}|$ by taking its nerve. In this paper we often abbreviate $|\mathcal{C}|$ to just $\mathcal{C}$, letting the application of the nerve be clear from context.

Finally, if $X$ is a spectrum then $\pi_{*} X$ always refers to the derived homotopy groups (that is, homotopy groups of a fibrant replacement). 
1.8.1 Acknowledgments The second author was partially supported by NSF Grant Number 0134938 and a Sloan Research Fellowship. The second author would also like to thank the Institut Mittag-Leffler (Djursholm, Sweden) for support during this work.

\section{Background on ring spectra}

In this section we give some of the basic constructions and properties of ring spectra which will be used throughout the paper.

\subsection{Postnikov sections}

Let $R$ be a connective, commutative ring spectrum. For any $R$-module $V$, we let $T_{R}(V)$ denote the tensor algebra on $V$. For any pointed simplicial set $K$, let $T_{R}(K)$ be shorthand for $T_{R}\left(R \wedge \Sigma^{\infty} K\right)$. Note that one has maps $T_{R}\left(\partial \Delta^{n}\right) \rightarrow T_{R}\left(\Delta^{n}\right)$, and these are cofibrations of $R$-algebras.

If $E$ is a cofibrant, connective $R$-algebra and

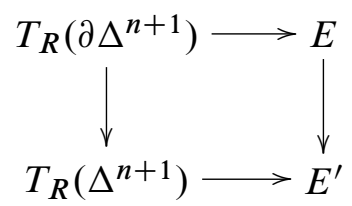

is a pushout diagram of $R$-algebras, one verifies that $\pi_{i}(E) \rightarrow \pi_{i}\left(E^{\prime}\right)$ is an isomorphism for $i \leq n-1$ (see Lemma A.3). Here we need that $E$ is cofibrant as an $R$-module (which follows from being cofibrant as an $R$-algebra, by [18, 4.1(3)]) to ensure that the pushout has the correct homotopy type-see the proof of Lemma A.3 for the details.

If $E$ is a cofibrant, connective $R$-algebra, let $P_{n}(E)$ be the result of applying the small object argument to $E$ with respect to the set of maps $T_{R}\left(\partial \Delta^{i}\right) \rightarrow T_{R}\left(\Delta^{i}\right)$ for all $i \geq n+2$ together with the generating trivial cofibrations for $R-\mathcal{A l g}$ from [13, 5.4.3] or $[18,4.1]$. This is similar to the functorial construction of a Postnikov section for differential graded algebras given in [7, 3.2]; see also [16, 5.1] for a detailed description of functorial Postnikov sections for symmetric spectra. One checks that $P_{n}(E)$ is fibrant, $\pi_{i} P_{n}(E)=0$ for $i>n$, and $\pi_{i} E \rightarrow \pi_{i} P_{n}(E)$ is an isomorphism for $i \leq n$. Also, one has natural maps $P_{n+1}(E) \rightarrow P_{n}(E)$ which are compatible with $E \rightarrow P_{n}(E)$ as $n$ varies.

Finally, if $E$ is a connective $R$-algebra then we will write $P_{n}(E)$ as shorthand for $P_{n}(c E)$, where $c E \rightarrow E$ is a fixed functorial cofibrant-replacement for $E$. Note that 
one does not have a map $E \rightarrow P_{n}(E)$ in this general case, only a zig-zag $E \stackrel{\sim}{\longleftarrow} E \rightarrow$ $P_{n}(c E)=P_{n}(E)$.

\subsection{Pushouts of ring spectra}

For the result below we will need to use relative homotopy groups $\pi_{*}(B, A)$ where $A \rightarrow B$ is a map of spectra. Note that we are not assuming that $A \rightarrow B$ is a cofibration, as is often done. What we mean by $\pi_{*}(B, A)$ is therefore $\pi_{*}(W, A)$, where we have functorially factored $A \rightarrow B$ as $A \hookrightarrow W \stackrel{\sim}{\longrightarrow} B$.

At many places in the paper we will use the following Blakers-Massey theorem for ring spectra:

2.3 Theorem Let $R$ be a connective, commutative ring spectrum and let $m, n \geq 1$. Suppose given a homotopy pushout square of $R$-algebras

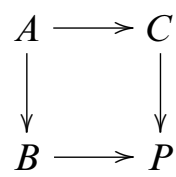

in which the following conditions hold:

(i) $A$ is connective.

(ii) $\pi_{i}(C, A)=0$ for $i<m$.

(iii) $\pi_{i}(B, A)=0$ for $i<n$.

Then $\pi_{i}(B, A) \rightarrow \pi_{i}(P, C)$ is an isomorphism for $i<m+n-1$ and a surjection for $i=m+n-1$. In particular, this means $\pi_{i}(P, C)=0$ for $i<n$, which implies $\pi_{i} C \rightarrow \pi_{i} P$ is an isomorphism for $i<n-1$ and a surjection for $i=n-1$.

This is an important result; however, we have not been able to find a proof of it in the literature. See Goerss and Hopkins [11, 2.3.13] or Baues [3, I.C.4.6], though, for full statements in related contexts. For completeness we have included a proof of Theorem 2.3 in Appendix A.

\subsection{Relative homotopy groups}

Let $A \rightarrow B$ be a map of $R$-algebras. We claim that $\pi_{*}(B, A)$ is in a natural way a bimodule over $\pi_{*} A$. To explain this, it suffices to assume that $A$ and $B$ are fibrant $R$-algebras, hence fibrant as spectra. Let $K \hookrightarrow L$ be a cofibration of pointed simplicial 
sets where $K$ is weakly equivalent to $\partial \Delta^{n}$ and $L$ is contractible. Then the relative homotopy group $\pi_{n}(B, A)$ may be described as equivalence classes of diagrams $\mathcal{D}$ of the form

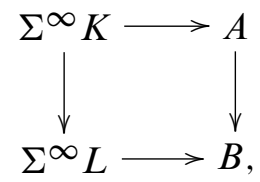

where two diagrams $\mathcal{D}$ and $\mathcal{D}^{\prime}$ are equivalent if there is a diagram

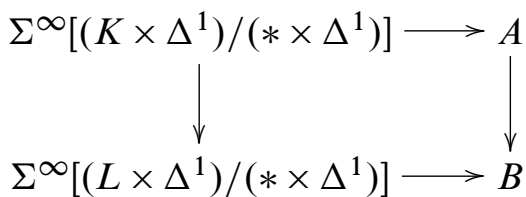

which restricts to $\mathcal{D}$ and $\mathcal{D}^{\prime}$ under the inclusions $\{0\} \hookrightarrow \Delta^{1}$ and $\{1\} \hookrightarrow \Delta^{1}$, respectively.

2.5 Remark To see why this description of $\pi_{n}(B, A)$ is valid, note that the above equivalence class of diagrams is precisely $\pi_{0}$ of the pullback of simplicial mapping spaces

$$
\operatorname{Map}\left(\Sigma^{\infty} K, A\right) \times_{\operatorname{Map}\left(\Sigma^{\infty} K, B\right)} \operatorname{Map}\left(\Sigma^{\infty} L, B\right) .
$$

Our assumptions on $A, B$, and $K \rightarrow L$ ensure that all the mapping spaces are fibrant and that $\operatorname{Map}\left(\Sigma^{\infty} L, B\right) \rightarrow \operatorname{Map}\left(\Sigma^{\infty} K, B\right)$ is a fibration. Since $L$ is contractible, $\operatorname{Map}\left(\Sigma^{\infty} L, B\right)$ is also contractible. So the above pullback is weakly equivalent to the homotopy fiber of $\operatorname{Map}\left(\Sigma^{\infty} K, A\right) \rightarrow \operatorname{Map}\left(\Sigma^{\infty} K, B\right)$, which is what we want.

Now suppose given an element $\alpha \in \pi_{n}(B, A)$, represented by a diagram $\mathcal{D}$ of the form

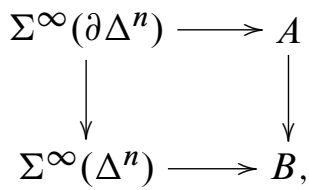

as above. Also assume given an element $\beta \in \pi_{k} A$ which is represented by a map $\Sigma^{\infty}\left(\partial \Delta^{k+1}\right) \rightarrow A$. Then one forms the new diagram

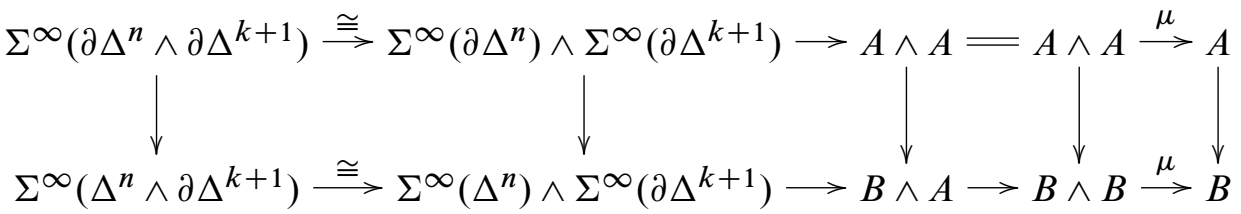

Algebraic 83 Geometric Topology, Volume 6 (2006) 
which represents a homotopy element of $\pi_{n+k}(B, A)$ (where we are taking $K=$ $\partial \Delta^{n} \wedge \partial \Delta^{k+1}$ and $\left.L=\Delta^{n} \wedge \partial \Delta^{k+1}\right)$. Here we would have been slightly better off if we were working with topological spaces rather than simplicial sets, as one can choose homeomorphisms $S^{n-1} \wedge S^{k} \cong S^{n+k-1}$ and $D^{n} \wedge S^{k} \cong D^{n+k}$; but everything works out simplicially as well, with only a little extra care.

We have just described a pairing $\pi_{n}(B, A) \times \pi_{k} A \rightarrow \pi_{n+k}(B, A)$, and one checks that this makes $\pi_{n}(B, A)$ into a right module over $\pi_{k} A$. A similar construction works for the left module structure, and the verification that this gives a bimodule is routine.

Note that in the long exact homotopy sequence of a pair, the connecting homomorphism $\partial: \pi_{n}(B, A) \rightarrow \pi_{n-1}(A)$ is a map of $\pi_{*} A$ bimodules. This is because $\partial$ sends a homotopy element represented by a diagram

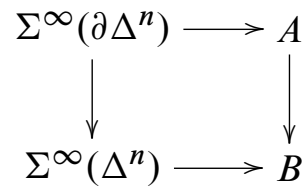

to the element $\Sigma^{\infty}\left(\partial \Delta^{n}\right) \rightarrow A$.

\section{$3 \quad k$-Invariants for ring spectra: an outline}

In this section we give a basic outline of how $k$-invariants work for ring spectra. A $k$-invariant gives rise to a Postnikov extension, and given a Postnikov extension we explain how to construct an associated $k$-invariant. These basic constructions will then be analyzed in a more sophisticated way later in the paper.

\subsection{Extensions of ring spectra}

We continue to assume that $R$ is a connective, commutative ring spectrum. Fix an $n \geq 1$. Let $C$ be a connective $R$-algebra such that $P_{n-1} C \simeq C$ and let $M$ be a $\pi_{0}(C)$-bimodule - that is, a $\left(\pi_{0} C\right) \otimes_{\pi_{0}(R)}\left(\pi_{0} C\right)^{o p}$-module. As mentioned in the introduction, we wish to consider ring spectra $Y$ together with a map $Y \rightarrow C$ such that

(i) $P_{n} Y \simeq Y$,

(ii) $P_{n-1} Y \rightarrow P_{n-1} C$ is a weak equivalence,

(iii) $\pi_{n}(Y) \cong M$ as $\pi_{0}(Y)$-bimodules (where $M$ becomes a $\pi_{0}(Y)$-bimodule via the isomorphism $\left.\pi_{0}(Y) \rightarrow \pi_{0}(C)\right)$. 
The map $Y \rightarrow C$ is called a Postnikov extension of $C$ of type $(M, n)$. Note that a particular choice of isomorphism $\pi_{n}(Y) \cong M$ is not part of the data.

Let $\mathcal{M}(C+(M, n))$ denote the category whose objects are such Postnikov extensions; here a map from $X \rightarrow C$ to $Y \rightarrow C$ is a weak equivalence $X \rightarrow Y$ making the evident triangle commute. We'll call this category the moduli space of Postnikov extensions of $C$ of type $(M, n)$.

If $\mathcal{C}$ is a category, we'll write $\pi_{0}(\mathcal{C})$ for the connected components of the nerve of $\mathcal{C}$. We wish to study $\pi_{0} \mathcal{M}(C+(M, n))$, as this will tell us how many "homotopically different" extensions of $C$ there are of type $(M, n)$.

3.2 Definition A functor $F: \mathcal{C} \rightarrow \mathcal{D}$ between categories will be called a weak equivalence if it induces a weak equivalence on the nerves. The functor $F$ will be called a homotopy equivalence if there is a functor $G: \mathcal{D} \rightarrow \mathcal{C}$ and zig-zags of natural transformations between $F \circ G$ and $\operatorname{Id}_{\mathcal{D}}$, and between $G \circ F$ and $\operatorname{Id}_{\mathcal{C}}$.

3.3 Proposition Suppose $C \rightarrow C^{\prime}$ is a weak equivalence of $R$-algebras. There is an evident functor $\rho: \mathcal{M}(C+(M, n)) \rightarrow \mathcal{M}\left(C^{\prime}+(M, n)\right)$ induced by composition, and this is a homotopy equivalence.

Proof We will construct a homotopy inverse. Given an object $X \rightarrow C^{\prime}$ of the category $\mathcal{M}\left(C^{\prime}+(M, n)\right)$, functorially factor this map as $X \stackrel{\sim}{\rightarrow} X_{1} \rightarrow C^{\prime}$. Letting $\phi(X)=C \times{ }_{C^{\prime}} X_{1}$, then $\phi(X) \rightarrow C$ is in $\mathcal{M}(C+(M, n))$, using right properness of $R-\mathcal{A l} g$. So this defines a functor $\phi: \mathcal{M}\left(C^{\prime}+(M, n)\right) \rightarrow \mathcal{M}(C+(M, n))$.

It is simple to check that there is a zig-zag of natural weak equivalences between the composite $\rho \circ \phi$ and the identity map, and the same for the other composite $\phi \circ \rho$.

By the above proposition, we can assume that $C$ is a cofibrant $R$-algebra when studying $\mathcal{M}(C+(M, n))$. We will always be clear when we are making this assumption, however.

\subsection{Bimodules}

By a $C$-bimodule we mean a left $\left(C \wedge{ }_{R} C^{o p}\right)$-module. As remarked in the introduction, there is a $C$-bimodule $H M$ satisfying $\pi_{i}(H M)=0$ for $i \neq 0$ and $\pi_{0}(H M) \cong M$ (as $\pi_{0}(C)$-bimodules). Moreover, a typical obstruction theory argument shows that any two such bimodules are weakly equivalent. We abbreviate $H M$ to just $M$ in the rest of the paper.

Algebraic 83 Geometric Topology, Volume 6 (2006) 
3.5 Remark Note that the notion of $C$-bimodule depends on more than just the homotopy type of $C$. For if $C \rightarrow C^{\prime}$ is a weak equivalence of $R$-algebras, the induced map $C \wedge_{R} C \rightarrow C^{\prime} \wedge_{R} C^{\prime}$ need not be a weak equivalence anymore. For this reason we will sometimes have to assume that $C$ is cofibrant as an $R$-module when dealing with bimodules.

\subsection{Extensions via pullbacks}

If $\mathcal{M}$ is a model category and $X \in \mathcal{M}$, let $\mathcal{M} / X$ denote the usual overcategory whose objects are maps $Y \rightarrow X$ in $\mathcal{M}$. Recall that $\mathcal{M}_{/ X}$ inherits a model structure from $\mathcal{M}$ in which a map from $Y \rightarrow X$ to $Y^{\prime} \rightarrow X$ is a cofibration (respectively fibration, weak equivalence) if and only if the map $Y \rightarrow Y^{\prime}$ is a cofibration (respectively fibration, weak equivalence) in $\mathcal{M}$.

We regard $C \vee \Sigma^{n+1} M$ as an object in $R-\mathcal{A l g} / C$ via the projection $C \vee \Sigma^{n+1} M \rightarrow C$. Note that $C \vee \Sigma^{n+1} M$ is actually a pointed object of $R-\mathcal{A l g} / C$, since it comes equipped with the evident inclusion $C \hookrightarrow C \vee \Sigma^{n+1} M$.

Suppose given a homotopy class in $\operatorname{Ho}(R-\mathcal{A l g} / C)\left(C, C \vee \Sigma^{n+1} M\right)$. This can be represented by a map

$$
\alpha: c C \rightarrow f\left(C \vee \Sigma^{n+1} M\right)
$$

where $c C$ is a cofibrant-replacement of $C$ and $f\left(C \vee \Sigma^{n+1} M\right)$ is a fibrant-replacement of $C \vee \Sigma^{n+1} M$ in $R-\mathcal{A l g} / C$. Consider the homotopy fiber of $\alpha$ in $R-\mathcal{A l g} / C$. This is the same as the homotopy pullback of

$$
c C \rightarrow f\left(C \vee \Sigma^{n+1} M\right) \leftarrow C
$$

in $R-\mathcal{A l g}$. To be precise, to form this homotopy pullback we functorially factor the maps as trivial cofibrations followed by fibrations

$$
c C \stackrel{\sim}{\hookrightarrow}(c C)^{\prime} \rightarrow f\left(C \vee \Sigma^{n+1} M\right), \quad C \stackrel{\sim}{\rightarrow} C^{\prime} \rightarrow f\left(C \vee \Sigma^{n+1} M\right)
$$

and then the homotopy pullback $Y$ is the pullback

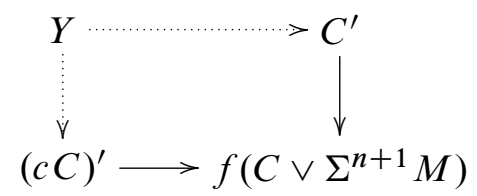

in $R-\mathcal{A l} g$. As pullbacks in $R-\mathcal{A l} g$ are the same as pullbacks in ordinary spectra, it is easy to analyze the homotopy groups of $Y$. One sees immediately that $\pi_{i}\left((c C)^{\prime}, Y\right)=0$ for $i \neq n+1$ and $\pi_{n+1}\left((c C)^{\prime}, Y\right) \cong M$. So $\pi_{i}(Y)=0$ for $i>n, \pi_{i} Y \rightarrow \pi_{i}(c C)^{\prime}$ is an isomorphism for $i<n$, and the map $\partial: \pi_{n+1}\left((c C)^{\prime}, Y\right) \rightarrow \pi_{n} Y$ is an isomorphism. 
By the remarks at the end of Section 2.4, $\partial$ is an isomorphism of $\pi_{0}(Y)$-bimodules. Moreover, the map $\pi_{n+1}\left((c C)^{\prime}, Y\right) \rightarrow \pi_{n+1}\left(f\left(C \vee \Sigma^{n+1} M\right), C\right)$ is an isomorphism of $\pi_{0}(Y)$-bimodules, and the codomain of this map is clearly isomorphic to $M$ as a bimodule. We have therefore shown that $Y$ is a Postnikov extension of $(c C)^{\prime}$ of type $(M, n)$. As we have a map $(c C)^{\prime} \rightarrow f\left(C \vee \Sigma^{n+1} M\right) \rightarrow C$ which is a weak equivalence, this is also a Postnikov extension of $C$ of type $(M, n)$.

The above remarks give us a function

$$
\text { PB: } \operatorname{Ho}(R-\mathcal{A l g} / C)\left(C, C \vee \Sigma^{n+1} M\right) \rightarrow \pi_{0} \mathcal{M}(C+(M, n)) \text {. }
$$

It is clearly not injective, for the following reason. An automorphism $\sigma: M \rightarrow M$ of $\pi_{0}(C)$-bimodules induces an automorphism of ring spectra $\sigma: C \vee \Sigma^{n+1} M \rightarrow$ $C \vee \Sigma^{n+1} M$. If a given homotopy class $\alpha \in \operatorname{Ho}(R-\mathcal{A l g} / C)\left(C, C \vee \Sigma^{n+1} M\right)$ is composed with this $\sigma$, it gives rise to a weakly equivalent pullback.

Let $\operatorname{Aut}(M)$ be the group of $\pi_{0}(C)$-bimodule automorphisms of $M$. One way to rephrase the above paragraph is to say that we have an action of $\operatorname{Aut}(M)$ on the set of homotopy classes we're considering, and we get an induced map

$$
\widetilde{\mathrm{PB}}:\left[\operatorname{Ho}(R-\mathcal{A l g} / C)\left(C, C \vee \Sigma^{n+1} M\right)\right] / \operatorname{Aut}(M) \rightarrow \pi_{0} \mathcal{M}(C+(M, n)) .
$$

Our main goal in this paper is to show that this map is an isomorphism. Along the way, however, we will actually describe the entire homotopy type of the space $\mathcal{M}(C+(M, n))$ as opposed to just $\pi_{0}$.

\section{$3.7 k$-Invariants}

Let $f: Y \rightarrow C$ be a Postnikov extension of type $(M, n)$. We wish to show that it's in the image of $\widetilde{\mathrm{PB}}$. We'll now give a rough outline of how to go about this, which will then be cleaned up in the later sections of the paper.

First of all, we can assume $Y$ is a cofibrant $R$-algebra (otherwise we replace it with one). Let $D$ be the homotopy pushout $C \amalg_{Y}^{h} C$ of $C \stackrel{f}{\stackrel{f}{\longrightarrow}} \stackrel{f}{\longrightarrow} C$ in $R-\mathcal{A l g} / C$. This means we factor $f$ as a cofibration followed by a trivial fibration

$$
Y \succ C^{\prime} \stackrel{\sim}{\longrightarrow} C
$$

and we let $D$ be the pushout

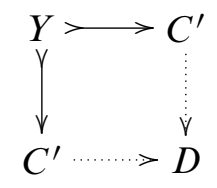

Algebraic 83 Geometric Topology, Volume 6 (2006) 
in $R-\mathcal{A l g}$. Note that there is a map $D \rightarrow C$ induced by the universal property of pushouts. Applying Theorem 2.3, we have that $\pi_{i}\left(C^{\prime}, Y\right) \rightarrow \pi_{i}\left(D, C^{\prime}\right)$ is an isomorphism for $i \leq 2 n$. It follows that $\pi_{i} C^{\prime} \rightarrow \pi_{i} D$ is an isomorphism for $i \leq n$, and $\pi_{n+1}\left(D, C^{\prime}\right) \cong M$ as $\pi_{0}\left(C^{\prime}\right)$-bimodules. (For the bimodule aspect of the last claim, one again uses the remarks in Section 2.4).

Let $E=P_{n+1} D$ (and note that $D$ is cofibrant, so that we have a natural map $D \rightarrow$ $\left.P_{n+1} D\right)$. We will later show that the map $C^{\prime} \rightarrow E$ is weakly equivalent in $R-\mathcal{A l g} / C$ to the standard inclusion $C \hookrightarrow C \vee \Sigma^{n+1} M$. This can easily be done by an obstruction theory argument (see also Remark 4.1). After choosing such a weak equivalence, we have that the composite map

$$
C^{\prime} \rightarrow D \rightarrow E
$$

represents an element of $\operatorname{Ho}\left(R-\mathcal{A} l g_{/ C}\right)\left(C, C \vee \Sigma^{n+1} M\right)$. We will show that choosing a different weak equivalence only affects this element up to the action of $\operatorname{Aut}(M)$, so that we have a well-defined invariant in

$$
\left[\operatorname{Ho}\left(R-\mathcal{A} l g_{/ C}\right)\left(C, C \vee \Sigma^{n+1} M\right)\right] / \operatorname{Aut}(M) .
$$

Some unpleasant checking is then required to verify that we have indeed produced an inverse to $\widetilde{\mathrm{PB}}$. To organize this checking, it helps to rephrase everything in terms of categories-this is what we do in the next section.

\section{Categories of $\boldsymbol{k}$-invariants and Eilenberg-Mac Lane objects}

Let $R, C, M$, and $n$ be as in the previous section. Our goal for the remainder of the paper is to analyze the homotopy type of the moduli space $\mathcal{N}(C+(M, n))$. To do this we need to introduce some auxiliary categories.

Define the category $\mathcal{E}_{C}(M, n)$ of $C$-Eilenberg-Mac Lane objects of type $(M, n)$ as follows. The objects of $\mathcal{E}_{C}(M, n)$ are maps $B \rightarrow E$ in $R-\mathcal{A l g} / C$ such that

(i) $B \rightarrow C$ is a weak equivalence,

(ii) $B \rightarrow E$ becomes a weak equivalence after applying $P_{n}$,

(iii) $P_{n+1} E \simeq E$,

(iv) $\pi_{n+1}(E) \cong M$ as $\pi_{0}(B)$-bimodules (where $M$ becomes a $\pi_{0}(B)$-bimodule via the isomorphism $\left.\pi_{0}(B) \rightarrow \pi_{0}(C)\right)$.

A map from $[B \rightarrow E]$ to $\left[B^{\prime} \rightarrow E^{\prime}\right]$ in this category consists of weak equivalences $B \rightarrow B^{\prime}$ and $E \rightarrow E^{\prime}$ in $R-\mathcal{A l g} / C$ making the evident square commute. 
4.1 Remark Although it is not entirely obvious, we will see later that every object of $\mathcal{E}_{C}(M, n)$ is weakly equivalent to $C \hookrightarrow C \vee \Sigma^{n+1} M$. This follows from Proposition 4.4(b) below, which shows that $\mathcal{E}_{C}(M, n)$ is connected.

Likewise, we define the category $\mathcal{K}_{C}(M, n)$ of generalized $k$-invariants for $C$ of type $(M, n)$. The objects of $\mathcal{K}_{C}(M, n)$ are pairs of maps $A \rightarrow E \leftarrow B$ in the category $R-\mathcal{A l g} / C$ such that

(i) $A \rightarrow C$ is a weak equivalence,

(ii) $B \rightarrow E$ is an object in $\mathcal{E}_{C}(M, n)$.

A map from $A \rightarrow E \leftarrow B$ to $A^{\prime} \rightarrow E^{\prime} \leftarrow B^{\prime}$ is a commutative diagram

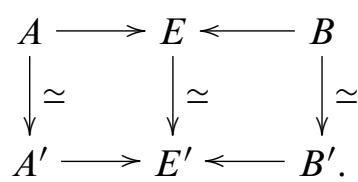

in $R-\mathcal{A l g} / C$ in which all the vertical maps are weak equivalences.

Note that there is a forgetful functor $\mathcal{K}_{C}(M, n) \rightarrow \mathcal{E}_{C}(M, n)$ which forgets the object $A$ and the map $A \rightarrow E$.

4.2 Proposition Suppose $C \rightarrow C^{\prime}$ is a weak equivalence of $R$-algebras. Then there are evident functors $\rho: \mathcal{K}_{C}(M, n) \rightarrow \mathcal{K}_{C^{\prime}}(M, n)$ and $\rho: \mathcal{E}_{C}(M, n) \rightarrow \mathcal{E}_{C^{\prime}}(M, n)$ induced by composition, and both are homotopy equivalences.

Proof We will prove the result for $\mathcal{K}_{C}(M, N)$ and leave the $\mathcal{E}_{C}(M, n)$ case to the reader. Given $A \rightarrow E \leftarrow B$ in $\mathcal{K}_{C^{\prime}}(M, n)$, produce functorial factorizations $A \stackrel{\sim}{\hookrightarrow} A^{\prime} \rightarrow C^{\prime}, B \stackrel{\sim}{\rightarrow} B^{\prime} \rightarrow C^{\prime}$, and $E \stackrel{\sim}{\hookrightarrow} E^{\prime} \rightarrow C^{\prime}$. So we have the diagram

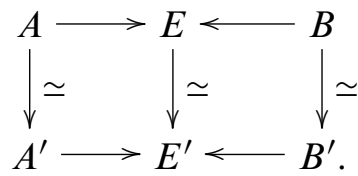

Define $\phi: \mathcal{K}_{C^{\prime}}(M, n) \rightarrow \mathcal{K}_{C}(M, n)$ by sending $A \rightarrow E \leftarrow B$ to the sequence of maps $A^{\prime} \times{ }_{C^{\prime}} C \rightarrow E^{\prime} \times{ }_{C^{\prime}} C \leftarrow B^{\prime} \times{ }_{C^{\prime}} C$. It follows by right-properness of $R-\mathcal{A l g}$ that this sequence indeed lies in $\mathcal{K}_{C}(M, n)$.

Just as in Proposition 3.3, it is simple to produce a zig-zag of natural weak equivalences between $\rho \circ \phi$ and the identity, and the same for $\phi \circ \rho$. 
Our next goal is to show that the category $\mathcal{K}_{C}(M, n)$ is weakly equivalent to the moduli space $\mathcal{N}(C+(M, n))$. First, observe that there is a functor $\mathrm{PB}_{C}: \mathcal{K}_{C}(M, n) \rightarrow$ $\mathcal{M}(C+(M, n))$ which sends $A \rightarrow E \leftarrow B$ to its homotopy pullback in $R-\mathcal{A l g} / C$. As in Section 3.6, whenever we talk about the "homotopy pullback" $A \times{ }_{E}^{h} B$ of a diagram $A \rightarrow E \leftarrow B$ we mean the pullback of $A^{\prime} \rightarrow E \leftarrow B^{\prime}$ where we have functorially factored $A \rightarrow E$ and $B \rightarrow E$ as trivial cofibrations followed by fibrations

$$
A \stackrel{\sim}{\mapsto} A^{\prime} \rightarrow E \quad \text { and } \quad B \stackrel{\sim}{\rightarrow} B^{\prime} \rightarrow E .
$$

Note that there is a natural map from the pullback of $A \rightarrow E \leftarrow B$ to its homotopy pullback.

To verify that the image of $\mathrm{PB}_{C}$ actually lands in $\mathcal{M}(C+(M, n))$, first recall that pullbacks of $R$-algebras are the same as pullbacks of spectra. This immediately verifies conditions (i) and (ii) in the definition of $\mathcal{M}(C+(M, n)$ ) (Section 3.1). For the third condition, let $P=A^{\prime} \times_{E} B^{\prime}$. Note that in the long exact homotopy sequence of a pair the connecting homomorphism $\pi_{n+1}\left(A^{\prime}, P\right) \rightarrow \pi_{n}(P)$ is an isomorphism since $P_{n-1} A^{\prime} \simeq A^{\prime}$, and in fact it is an isomorphism of $\pi_{0}(P)$-bimodules by the discussion in Section 2.4. But we also have an isomorphism of $\pi_{0}(P)$-bimodules $\pi_{n+1}\left(A^{\prime}, P\right) \rightarrow \pi_{n+1}\left(E, B^{\prime}\right)$, as well as an isomorphism $\pi_{n+1}(E) \rightarrow \pi_{n+1}\left(E, B^{\prime}\right)$ of $\pi_{0}\left(B^{\prime}\right)$-bimodules. For the fact that these are bimodule maps, we again refer to Section 2.4. Since $\pi_{n+1}\left(E, B^{\prime}\right) \cong \pi_{n+1}(E, B) \cong M$, we find that $\pi_{n}(P)$ is isomorphic to $M$ as $\pi_{0}(P)$-bimodules.

4.3 Proposition The functor $\mathrm{PB}_{C}: \mathcal{K}_{C}(M, n) \rightarrow \mathcal{M}(C+(M, n))$ is a weak equivalence.

The proof is somewhat long, and will be given in Section 5. The basic idea is to try to construct a homotopy inverse functor $k: \mathcal{M}(C+(M, n)) \rightarrow \mathcal{K}_{C}(M, n)$. This will be our generalized k-invariant. Given an $X \rightarrow C$ in $\mathcal{M}(C+(M, n))$ such that $X$ is cofibrant, construct a homotopy pushout

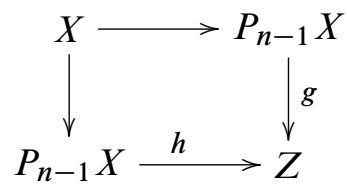

and consider the maps

$$
P_{n-1} X \stackrel{h}{\longrightarrow} P_{n+1} Z \stackrel{g}{\longleftarrow} P_{n-1} X .
$$


One can check that this gives an element $k(X)$ in $\mathcal{K}_{P_{n+1}\left(P_{n-1} C\right)}(M, n)$. This is almost an element of $\mathcal{K}_{C}(M, n)$ since $P_{n+1}\left(P_{n-1} C\right) \simeq C$. Some care is required in getting around this small difference, and this is part of what is accomplished in Section 5.

The above proposition reduces the problem of studying $\mathcal{M}(C+(M, n))$ to that studying $\mathcal{K}_{C}(M, n)$. We do this by analyzing the forgetful functor $\mathcal{K}_{C}(M, n) \rightarrow$ $\mathcal{E}_{C}(M, n)$. We will prove the following in Section 7:

\subsection{Proposition}

(a) There is a homotopy fiber sequence of spaces

$$
\underline{R-\mathcal{A} l g_{/ C}}\left(C, C \vee \Sigma^{n+1} M\right) \rightarrow\left|\mathcal{K}_{C}(M, n)\right| \rightarrow\left|\mathcal{E}_{C}(M, n)\right|,
$$

where the first term denotes the homotopy function complex in the model category $R-\mathcal{A l g} / C$.

(b) There is a weak equivalence of spaces $\left|\mathcal{E}_{C}(M, n)\right| \simeq B \operatorname{Aut}(M)$ where $\operatorname{Aut}(M)$ is the group of automorphisms of $M$ as a $\pi_{0}(C)$-bimodule.

Part (a) is basically a routine "moduli space" problem, of the type considered in [4, Section 2]. Part (b) involves similar techniques but also requires some careful manipulations of ring spectra.

\section{The moduli space of $k$-invariants}

In this section we will prove Proposition 4.3. As mentioned above, our first hope would be for a generalized k-invariant functor $k: \mathcal{N}(C+(M, n)) \rightarrow \mathcal{K}_{C}(M, n)$ to provide a homotopy inverse for $\mathrm{PB}_{C}: \mathcal{K}_{C}(M, n) \rightarrow \mathcal{M}(C+(M, n))$. This doesn't quite work out. Instead, we restrict to the case where $C$ is cofibrant and construct a functor $k: \mathcal{M}(C+(M, n)) \rightarrow \mathcal{K}_{C^{\prime}}(M, n)$ where $C^{\prime}=P_{n+1}\left(P_{n-1} C\right)$. We then use this to show that $\mathrm{PB}_{C}$ is a weak equivalence.

For the rest of this section we assume that $C$ is a cofibrant $R$-algebra. Throughout the following, let $C^{\prime}=P_{n+1}\left(P_{n-1} C\right)$. Note that since we are using the functor $P_{n+1}$ coming from the small object argument described in Section 2.1, the map $P_{n-1} C \rightarrow P_{n+1}\left(P_{n-1} C\right)$ is only a weak equivalence and not an isomorphism. Next we define $\mathcal{D}(C, M, n)$, a category of diagrams which will be useful in defining a generalized k-invariant functor $k: \mathcal{M}(C+(M, n)) \rightarrow \mathcal{K}_{C^{\prime}}(M, n)$. 
The objects of $\mathcal{D}(C, M, n)$ are the commutative diagrams $D$ of the form

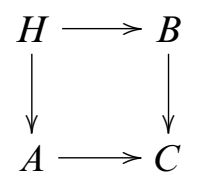

in which $A \rightarrow C$ and $B \rightarrow C$ are weak equivalences after applying $P_{n-1}$, and $H \rightarrow C$ lies in $\mathcal{M}(C+(M, n))$. The morphisms in $\mathcal{D}(C, M, n)$ are the maps of commuting diagrams. Using the weak equivalence $C \rightarrow P_{n-1} C \rightarrow P_{n+1}\left(P_{n-1} C\right)=C^{\prime}$, we will produce a diagram

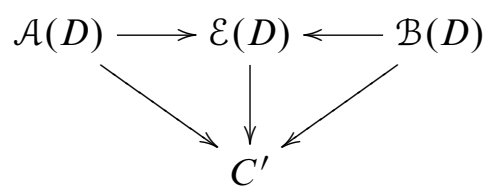

which is functorial in $\mathcal{D}(C, M, n)$ and has the following properties:

(1) The diagram $\mathcal{A}(D) \rightarrow \mathcal{E}(D) \leftarrow \mathcal{B}(D)$ lies in $\mathcal{K}_{C^{\prime}}(M, n)$, where we have used the weak equivalence $C \rightarrow C^{\prime}$ to make $M$ a bimodule over $\pi_{0}\left(C^{\prime}\right)$.

(2) There is a natural zig-zag of weak equivalences in $R-\mathcal{A l g} / C^{\prime}$ between $H$ and the homotopy pullback of $\mathcal{A}(D) \rightarrow \mathcal{E}(D) \leftarrow \mathcal{B}(D)$.

(3) If $A \rightarrow E \leftarrow B$ is an object in $\mathcal{K}_{C}(M, n)$ in which the indicated maps are fibrations, and $D$ is the diagram

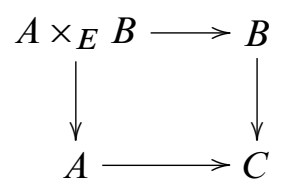

then there is a natural zig-zag of weak equivalences between $A \rightarrow E \leftarrow B$ and $\mathcal{A}(D) \rightarrow \mathcal{E}(D) \leftarrow \mathcal{B}(D)$ in $R-\mathcal{A l g} / C^{\prime}$.

(4) Suppose $D^{\prime}$ is another diagram

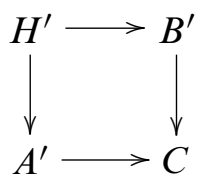

in $\mathcal{D}(C, M, n)$, and assume there is a map of diagrams $D \rightarrow D^{\prime}$ which is the identity on $C$, a weak equivalence on $H \rightarrow H^{\prime}$ and on $B \rightarrow B^{\prime}$, and a weak equivalence after applying $P_{n-1}$ to $A \rightarrow A^{\prime}$. Then the induced maps $\mathcal{A}(D) \rightarrow \mathcal{A}\left(D^{\prime}\right), \mathcal{E}(D) \rightarrow \mathcal{E}\left(D^{\prime}\right)$, and $\mathcal{B}(D) \rightarrow \mathcal{B}\left(D^{\prime}\right)$ are weak equivalences. 
In a moment we will explain how to construct $\mathcal{A}(D), \mathcal{E}(D)$, and $\mathcal{B}(D)$, and we will verify the above properties. But first we show how this implies what we want.

Proof of Proposition 4.3 Using Proposition 3.3 and Proposition 4.2, it suffices to analyze the case when $C$ is a cofibrant $R$-algebra.

Suppose given $X \rightarrow C$ in $\mathcal{M}(C+(M, n))$. We define $k(X) \in \mathcal{K}_{C^{\prime}}(M, n)$ to be $\mathcal{A}(D) \rightarrow \mathcal{E}(D) \leftarrow \mathcal{B}(D)$ where $D$ is the diagram

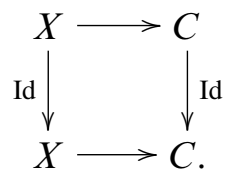

We think of $k(X)$ as the generalized $k$-invariant of $X$. It gives a functor $k$ from $\mathcal{M}(C+(M, n))$ to $\mathcal{K}_{C^{\prime}}(M, n)$.

We now have the following (noncommutative) diagram of functors:

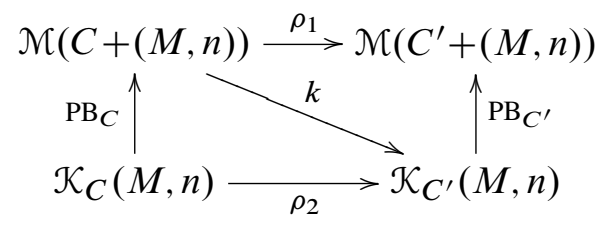

The maps labelled $\rho_{1}$ and $\rho_{2}$ are known to be weak equivalences, by Proposition 3.3 and Proposition 4.2. If we can show that there is a zig-zag of natural transformations between $\rho_{1}$ and the composite $\mathrm{PB}_{C^{\prime}} \circ k$, as well as between $\rho_{2}$ and the composite $k \circ \mathrm{PB}_{C}$, it will follow that all maps in the diagram induce isomorphisms on the homotopy groups of the nerves—so all the maps will be weak equivalences.

Now, property (2) says precisely that there is a zig-zag of natural weak equivalences between $\mathrm{PB}_{C^{\prime}} \circ k$ and $\rho_{1}$. So we consider the other composite. Let $A \rightarrow E \leftarrow B$ be an object in $\mathcal{K}_{C}(M, n)$. Functorially factor $A \rightarrow E$ and $B \rightarrow E$ as

$$
A \stackrel{\sim}{\hookrightarrow} A^{\prime} \rightarrow E \quad \text { and } \quad B \stackrel{\sim}{\curvearrowleft} B^{\prime} \rightarrow E
$$

and let $H=A^{\prime} \times_{E} B^{\prime}$. So $\operatorname{PB}_{C}(A \rightarrow E \leftarrow B)=H$. Let $D_{1}, D_{2}$, and $D_{3}$ be the following three squares:
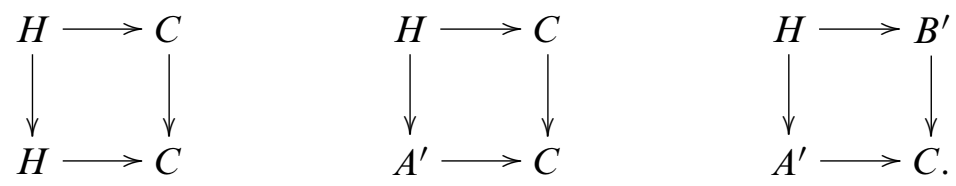

Algebraic 83 Geometric Topology, Volume 6 (2006) 
Note that there are natural transformations $D_{1} \rightarrow D_{2}$ and $D_{3} \rightarrow D_{2}$. By property (4), we get the following chain of equivalences:

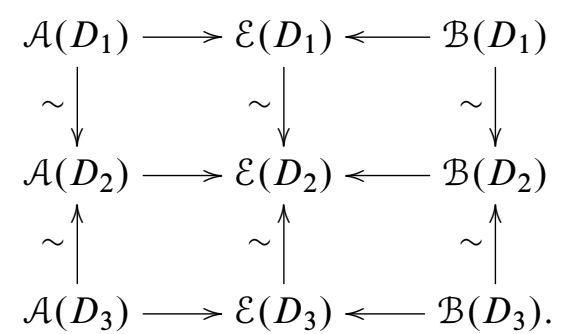

Note that the top row is $k(H)=k\left(\mathrm{~PB}_{C}(A \rightarrow E \leftarrow B)\right)$. Using property (3), there is a natural zig-zag of weak equivalences between the last row and the diagram $A^{\prime} \rightarrow$ $E \leftarrow B^{\prime}$, which in turn is weakly equivalent to $A \rightarrow E \leftarrow B$. So we have established our zig-zag of natural transformations between $k \circ \mathrm{PB}_{C}$ and $\rho_{2}$. This completes the proof.

Finally we are reduced to doing some actual work: we must construct the functors $\mathcal{A}$, $\mathcal{E}$, and $\mathcal{B}$. Suppose given a diagram $D$ in $\mathcal{D}(C, M, n)$ of the form

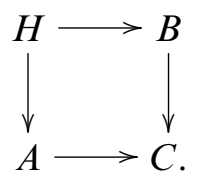

Recall that $A \rightarrow C$ and $B \rightarrow C$ are weak equivalences after applying $P_{n-1}$, and $H \rightarrow C$ lies in $\mathcal{M}(C+(M, n))$. Let $c X \stackrel{\sim}{\longrightarrow} X$ be a functorial cofibrant-replacement in $R-\mathcal{A l g}$. Consider the composites $c H \rightarrow P_{n-1}(c H) \rightarrow P_{n-1}(c A)$ and $c H \rightarrow$ $P_{n-1}(c H) \rightarrow P_{n-1}(c B)$ : functorially factor them as

$$
c H \longmapsto S A \stackrel{\sim}{\longrightarrow} P_{n-1}(c A) \quad \text { and } \quad c H \succ S B \stackrel{\sim}{\longrightarrow} P_{n-1}(c B) .
$$

We obtain a diagram

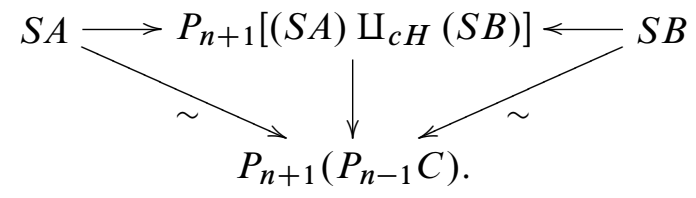

We let $\mathcal{A}(D)=S A, \mathcal{E}(D)=P_{n+1}\left[(S A) \amalg_{c H}(S B)\right], \mathcal{B}(D)=S B$, and $C^{\prime}=$ $P_{n+1}\left(P_{n-1} C\right)$.

Notice the following:

- Property (4) follows immediately from our definitions. 
- There is a natural map from $c H$ into the pullback $\mathcal{A}(D) \times \varepsilon(D) \mathcal{B}(D)$, and of course a natural map from the pullback to the homotopy pullback. This gives a natural zig-zag

$$
H \stackrel{\sim}{\longleftarrow} H \rightarrow \mathcal{A}(D) \times_{\mathcal{E}(D)}^{h} \mathcal{B}(D)
$$

in $R-\mathcal{A l g} / C^{\prime}$.

In order to check the remaining properties we will need the following two lemmas.

5.1 Lemma Fix $n \geq 1$. Let $W$ be a connective $R$-algebra satisfying $P_{n-1} W \simeq W$, and let $M$ be a $\pi_{0}(W)$-bimodule. Let $A \leftarrow X \rightarrow B$ be maps in $R-\mathcal{A l g} / W$ where $A \rightarrow W$ and $B \rightarrow W$ are weak equivalences and $X \rightarrow W$ is in $\mathcal{M}(W+(M, n))$. Let $P$ denote the homotopy pushout $A \amalg_{X}^{h} B$. Then $\pi_{n+1} P$ is isomorphic to $\pi_{n} X$ as a $\pi_{0}(X)$-bimodule.

Proof Consider the map $f: \pi_{n+1}(A, X) \rightarrow \pi_{n+1}(P, B)$, which is an isomorphism by Theorem 2.3. This is readily seen to be a map of $\pi_{0}(X)$-bimodules, using the observations from Section 2.4. Here we regard $\pi_{n+1}(P, B)$ as a $\pi_{0}(X)$-bimodule via the map of $\pi_{0}(R)$-algebras $\pi_{0}(X) \rightarrow \pi_{0}(B)$. The map $\pi_{n+1}(P) \rightarrow \pi_{n+1}(P, B)$ is a map of $\pi_{0}(B)$-bimodules (and hence $\pi_{0}(X)$-bimodules, by restriction) which is an isomorphism by our assumptions on $B$.

Finally, the connecting homomorphism $\pi_{n+1}(A, X) \rightarrow \pi_{n}(X)$ is a map of $\pi_{0}(X)-$ bimodules which is an isomorphism by our assumptions on $X$ and $A$. So we have established that $\pi_{n+1}(P) \cong \pi_{n}(X)$ as $\pi_{0}(X)$-bimodules.

5.2 Lemma Let $n \geq 1$ and let $W, M$ be as in the previous lemma.

(a) Suppose that $A \rightarrow E \leftarrow B$ is in $\mathcal{K}_{W}(M, n)$ (where the indicated maps are fibrations), and let $H=A \times_{E} B$. Then the induced map $A \amalg_{H}^{h} B \rightarrow E$ becomes a weak equivalence after applying $P_{n+1}$.

(b) Suppose we are given cofibrations $A \hookleftarrow X \succ B$ in $R-\mathcal{A l g} / W$ such that both $A \rightarrow W$ and $B \rightarrow W$ are weak equivalences, and where $P_{n} X \simeq X$. Also assume both $P_{n-1} X \rightarrow P_{n-1} A$ and $P_{n-1} X \rightarrow P_{n-1} B$ are weak equivalences. Then the diagram $A \rightarrow P_{n+1}\left(A \amalg_{X} B\right) \leftarrow B$ lies in $\mathcal{K}_{P_{n+1} W}\left(\pi_{n} X, n\right)$.

(c) Again suppose the given cofibrations $A \longleftarrow X \succ B$ in $R-\mathcal{A l g} / W$ satisfy the same hypotheses as in (b). Let $X^{\prime}$ be the homotopy pullback of $A \rightarrow$ $P_{n+1}\left(A \amalg_{X} B\right) \leftarrow B$. Then the induced map $X \rightarrow X^{\prime}$ is a weak equivalence. 
Proof The statements in (a) and (c) follow directly from Theorem 2.3. One should note that pullbacks (and homotopy pullbacks) in the category of $R$-algebras are the same as those in the category of symmetric spectra.

For the statement in (b), one uses Theorem 2.3 together with Lemma 5.1 above.

Proof of Properties (1)-(3) Properties (1) and (2) follow directly from Lemma 5.2 parts (b) and (c), respectively. So we turn to property (3).

Let $A \rightarrow E \leftarrow B$ be an object in $\mathcal{K}_{C}(M, n)$, and let $H$ be the pullback $A \times_{E} B$. Let $\mathcal{D}$ be the diagram

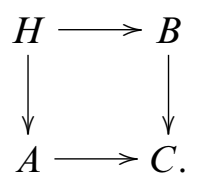

Functorially factor the maps $c H \rightarrow c A$ and $c H \rightarrow c B$ as $c H \hookrightarrow S^{\prime} A \stackrel{\sim}{\longrightarrow} A$ and $c H \succ S^{\prime} B \stackrel{\sim}{\longrightarrow} c B$. Note that one gets induced maps $S^{\prime} A \rightarrow S A$ and $S^{\prime} B \rightarrow S B$ (where $S A$ and $S B$ appeared in our construction of $\mathcal{A}(D)$, etc.), and these maps are weak equivalences. Let $\mathcal{E}^{\prime}(D)=P_{n+1}\left(S^{\prime} A \amalg_{c H} S^{\prime} B\right)$, so that there is an induced map $\mathcal{E}^{\prime}(D) \rightarrow \mathcal{E}(D)$.

Notice that we have a map $S^{\prime} A \amalg_{c H} S^{\prime} B \rightarrow c A \amalg_{c H} c B \rightarrow c E$, and therefore get an induced map $\mathcal{E}^{\prime}(D) \rightarrow P_{n+1}(c E)$. This is a weak equivalence by Lemma 5.2(a). Now we have the following:

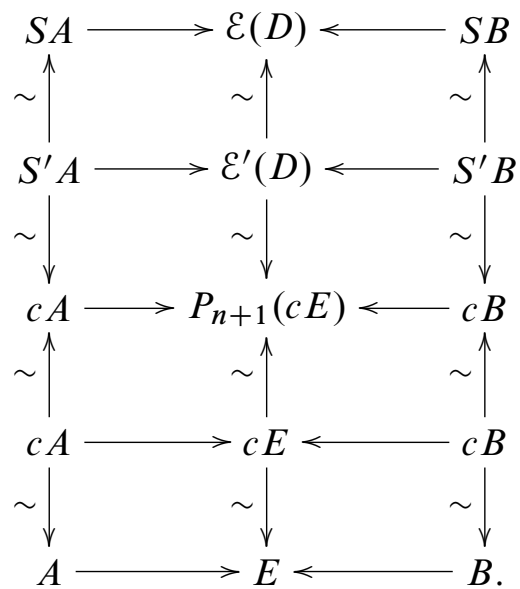

So we have obtained a natural zig-zag of weak equivalences between the diagrams $\mathcal{A}(D) \rightarrow \mathcal{E}(D) \leftarrow \mathcal{B}(D)$ and $A \rightarrow E \leftarrow B$ 


\section{Nonunital algebras}

Before proceeding further with our main results we need to develop a little machinery. This concerns nonunital $C$-algebras and their relations to $C$-bimodules. We first discuss a model structure on nonunital $C$-algebras. Then we define an "indecomposables" functor from nonunital $C$-algebras to $C$-bimodules and study its interaction with Postnikov stages. All of this basic machinery has been heavily influenced by Basterra [1].

Let $R$ be a commutative $S$-algebra and $C$ an $R$-algebra. Define a nonunital $C-$ algebra to be a nonunital monoid in the category of $C$-bimodules, that is, an algebra over the monad

$$
\widetilde{T}_{C}(M)=M \amalg\left(M \wedge_{C} M\right) \amalg\left(M \wedge_{C} M \wedge_{C} M\right) \amalg \cdots
$$

in C-bimod. Let $\mathcal{N} o n U_{C}$ denote the category of nonunital $C$-algebras.

A map of nonunital $C$-algebras is defined to be a fibration or a weak equivalence if the underlying map in $C$-bimod (or $R$-Mod) is a fibration or a weak equivalence. A map is then a cofibration if it has the left lifting property with respect to all trivial fibrations. Below we will use [18] to verify that this gives a model structure on $\mathcal{N} o n U_{C}$.

First recall that $\mathrm{C}$-bimod is just another name for the category $C \wedge_{R} C^{o p}-\mathrm{Mod}$. The model structure on $R$-modules lifts to a model structure on $C$-bimodules [18, 4.1]. Let $F_{C}: R$-Mod $\rightarrow$ C-bimod be the left adjoint to the forgetful functor C-bimod $\rightarrow$ $R$-Mod. If $j: K \rightarrow L$ is a generating trivial cofibration in $R$-Mod, then the generating trivial cofibrations in C-bimod are of the form $F_{C}(j): C \wedge_{R} K \wedge_{R} C \rightarrow C \wedge_{R} L \wedge_{R} C$.

6.1 Theorem The above notions of cofibration, fibration, and weak equivalence form a cofibrantly generated model category structure on $\mathcal{N o n} U_{C}$. The generating cofibrations and trivial cofibrations are of the form $\widetilde{T}_{C}\left(F_{C}(K)\right) \rightarrow \widetilde{T}_{C}\left(F_{C}(L)\right)$ where $K \rightarrow L$ is a generating cofibration or trivial cofibration in $R-$ Mod.

Proof To establish the model structure on $\mathcal{N o n U}_{C}$ we modify the arguments for unital monoids in [18, 6.2]. The argument in [18] is mostly formal except for one key step. For us, this step is to show that given a generating trivial cofibration $K \rightarrow L$ in C-bimod, the pushout in $\mathcal{N} o n U_{C}$ of the diagram

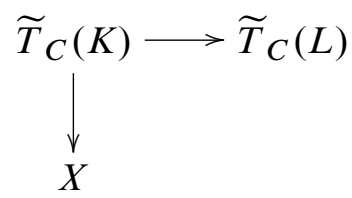

Algebraic 83 Geometric Topology, Volume 6 (2006) 
is the colimit $P=\operatorname{colim} P_{n}$ in $C$-bimod of a sequence

$$
X=P_{0} \rightarrow P_{1} \rightarrow \cdots \rightarrow P_{n} \rightarrow \cdots
$$

where $P_{n}$ is obtained from $P_{n-1}$ by a pushout in C-bimod. We then show that the monoid axiom implies that $X=P_{0} \rightarrow P$ is a weak equivalence. From this it follows directly from $[18,2.3(1)]$ that the given model structure exists on $\mathcal{N} o n U_{C}$.

To construct $P_{n}$ from $P_{n-1}$, we replace the functor $W$ in $[18,6.2]$ by a functor

$$
W: \mathcal{P}(\underline{n}) \rightarrow C-\text { bimod }
$$

where $\mathcal{P}(\underline{n})$ is the poset category of subsets of $\underline{n}=\{1,2, \cdots, n\}$ and inclusions. For $S \subseteq \underline{n}$, define

$$
W(S)=(C \vee X) \wedge_{C} B_{1} \wedge_{C}(C \vee X) \wedge_{C} B_{2} \wedge_{C} \cdots \wedge_{C} B_{n} \wedge_{C}(C \vee X)
$$

with

$$
B_{i}= \begin{cases}K & \text { if } i \notin S \\ L & \text { if } i \in S .\end{cases}
$$

Let $Q_{n}$ be the colimit of $W(S)$ over $\mathcal{P}(\underline{n})-\underline{n}$ (that is, the proper subsets of $\underline{n}$ ). As in [18, 6.2], one has maps $Q_{n} \rightarrow P_{n-1}$ and $Q_{n} \rightarrow W(\underline{n})$ and defines $P_{n}$ as the following pushout in $C$-bimod

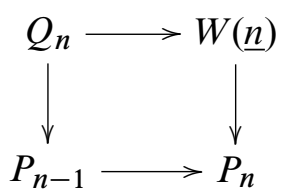

Set $P=$ colim $P_{n}$, the colimit in $C$-bimod. Arguments analogous to those in [18, 6.2] show that $P$ is naturally a nonunital $C$-algebra and has the universal property of the pushout of $X \leftarrow \widetilde{T}_{C}(K) \rightarrow \widetilde{T}_{C}(L)$ in nonunital $C$-algebras.

We next show that the monoid axiom for $R$-Mod implies that each map $P_{n-1} \rightarrow P_{n}$ is a weak equivalence whenever $K \rightarrow L$ is a generating trivial cofibration in $C$-bimod. These generating trivial cofibrations are of the form $F_{C}\left(K^{\prime}\right) \rightarrow F_{C}\left(L^{\prime}\right)$ where $K^{\prime} \rightarrow L^{\prime}$ is a generating trivial cofibration in $R-$ Mod.

Since pushouts in $C$-bimod are created in $R$-Mod which is symmetric monoidal, we consider the pushouts defining $P_{n}$ in $R$-Mod. Replacing $K \rightarrow L$ by $F_{C}\left(K^{\prime}\right) \rightarrow$ $F_{C}\left(L^{\prime}\right)$, we see that $Q_{n} \rightarrow W(\underline{n})$ is isomorphic to

$$
Q_{n}^{\prime} \wedge_{R}(C \vee X)^{\wedge_{R}(n)} \rightarrow\left(L^{\prime}\right)^{\wedge_{R} n} \wedge_{R}(C \vee X)^{\wedge_{R}(n)}
$$


where $Q_{n}^{\prime} \rightarrow\left(L^{\prime}\right)^{\wedge}{ }_{R}^{n}$ is the $n$-fold box product of $K^{\prime} \rightarrow L^{\prime}$. The pushout product axiom implies that $Q_{n}^{\prime} \rightarrow\left(L^{\prime}\right)^{\wedge}{ }_{R}^{n}$ is a trivial cofibration. The monoid axiom then implies that the pushouts $P_{n-1} \rightarrow P_{n}$ are weak equivalences and $X=P_{0} \rightarrow \operatorname{colim} P_{n}$ is a weak equivalence.

There is a functor $K: \mathcal{N} o n U_{C} \rightarrow(C \downarrow R-\mathcal{A l g} \downarrow C)$ which takes a nonunital algebra $N$ to $K(N)=(C \stackrel{\eta}{\rightarrow} C \vee N \stackrel{\pi}{\longleftarrow} C)$ where $\eta$ and $\pi$ are the obvious inclusion and projection. This has a right adjoint

$$
I:(C \downarrow R-\mathcal{A l g} \downarrow C) \rightarrow \mathcal{N o n} U_{C}
$$

called the augmentation ideal functor. The functor $I$ sends $(C \rightarrow X \rightarrow C)$ to the fiber of $X \rightarrow C$.

6.2 Proposition The functors $(K, I)$ form a Quillen equivalence

$$
K: \mathcal{N o n} U_{C} \stackrel{\sim}{\longrightarrow}(C \downarrow R-\mathcal{A l g} \downarrow C) .
$$

Proof The same statement for commutative ring spectra is proved in Basterra [1, 2.2]. The proof works verbatim in the noncommutative case; see also Basterra and Mandell [2, Theorem 8.6] for a vast generalization.

\subsection{Indecomposables}

Our next task is to compare nonunital $C$-algebras with $C$-bimodules. The indecomposables functor $Q: \mathcal{N} o n U_{C} \rightarrow$ C-bimod takes $X$ in $\mathcal{N} o n U_{C}$ to the pushout of $* \leftarrow X \wedge X \rightarrow X$. Its right adjoint $Z: \mathrm{C}$-bimod $\rightarrow \mathcal{N}$ on $U_{C}$ sends a bimodule to itself equipped with the zero product.

6.4 Proposition The functors $Q$ and $Z$ form a Quillen pair:

$$
\mathcal{N o n} U_{C} \underset{Z}{\stackrel{Q}{\rightleftarrows}} C \text {-bimod. }
$$

Proof The functor $Z$ obviously preserves fibrations and trivial fibrations. Again, see $[1,3.1]$ and $[2$, Proposition 8.7$]$ for similar statements.

If $N$ is a $C$-bimodule, it is easy to see that $Q(Z N) \cong N$. When we consider the derived functors $Q(\underline{Z} N)$ the situation changes, however. Here we must take a cofibrant replacement of $\overline{Z N}$ before applying $Q$. It turns out that $\underline{Q}(\underline{Z} N)$ typically has nonzero homotopy groups in infinitely many dimensions, even if $N$ did not. 
If $n \geq 1$ and $N$ has no homotopy groups in dimensions smaller than $n$, then the same turns out to be true for $Q(\underline{Z} N)$. Moreover, the $n$-th homotopy group of $\underline{Q}(\underline{Z} N)$ is easy to analyze, and it is the same as that of $N$. This is the content of Proposition 6.5 below. We remark that this proposition bears some relation to [1, 8.2].

Before stating the proposition we need a couple of pieces of new notation. We'll use $c$ and $f$ to denote cofibrant- and fibrant-replacement functors in a model category, and we leave it to the reader to decide from context which model category the replacements are taking place in. In the statement of the proposition below, for instance, the $c$ is being applied in $\mathcal{N} o n U_{C}$ and the $f$ 's are being applied in $C$-bimod.

Also, note that the $P_{n}$ 's in the statement of the proposition refer to Postnikov sections in the category of $C$-bimodules. These are constructed analogously to those for ring spectra, but here one forms pushouts with respect to the maps $\left(C \wedge{ }_{R} C^{o p}\right) \wedge \partial \Delta^{i} \rightarrow$ $\left(C \wedge{ }_{R} C^{o p}\right) \wedge \Delta^{i}$ for $i>n+1$.

6.5 Proposition Fix $n \geq 1$, and let $N$ be a $C$-bimodule such that $\pi_{i}(N)=0$ for $i<n$. There is a natural weak equivalence of $C$-bimodules

$$
P_{n}[Q c Z(f N)] \rightarrow P_{n}(f N) .
$$

Proof First note that there are natural maps $Q c Z(f N) \rightarrow Q Z(f N) \rightarrow f N$. Applying $P_{n}$ to this composite gives the map in the statement of the proposition. Call this map $g$.

Recall that $\widetilde{T}_{C}: C$-bimod $\rightarrow \mathcal{N} o n U_{C}$ is the left adjoint of the forgetful functor. Note that this is a left Quillen functor, and that there are natural isomorphisms $Q \widetilde{T}_{C}(W) \cong$ $W$ by an easy adjointness argument.

If $K$ is a spectrum, write $F K$ as shorthand for $\left(C \wedge_{R} C^{o p}\right) \wedge K$. This is the free $C$-bimodule generated by $K$.

Write $\mathbb{P}_{n}$ for the Postnikov section functor in the category $\mathcal{N} o n U_{C}$. The construction is the same as for ring spectra, but in this case we form pushouts with respect to the maps $\widetilde{T}_{C}\left(F \partial \Delta^{i}\right) \rightarrow \widetilde{T}_{C}\left(F \Delta^{i}\right)$ for $i>n+1$. Using that $Q$ is a left adjoint and therefore preserves pushouts and colimits, and that $Q \widetilde{T}_{C}(W) \cong W$, one can show that $Q\left(\mathbb{P}_{n} X\right)$ is obtained from $Q X$ by forming pushouts with respect to $F\left(\partial \Delta^{i}\right) \rightarrow F\left(\Delta^{i}\right)$ for $i>n+1$. Given the description above of Postnikov sections for $C$-bimodules, this implies that the map $Q X \rightarrow Q\left(\mathbb{P}_{n} X\right)$ induces a weak equivalence

$$
P_{n}[Q X] \rightarrow P_{n}\left[Q \mathbb{P}_{n} X\right] .
$$

Without loss of generality we may assume that $N$ is a cofibrant $C$-bimodule. Consider the natural map of nonunital algebras $\widetilde{T}_{C}(N) \rightarrow Z(N)$, adjoint to the isomorphism 
$N \rightarrow U Z(N)$ where $U: \mathcal{N o n} U_{C} \rightarrow C$-bimod is the forgetful functor. Using our hypothesis on $N$, one finds that $N \wedge_{C} N \wedge_{C} \cdots \wedge_{C} N$ doesn't have any homotopy groups in dimensions less than $2 n$ as long as there are at least two smash factors of $N$. It follows immediately that the induced map $\mathbb{P}_{n}\left[\widetilde{T}_{C}(N)\right] \rightarrow \mathbb{P}_{n}(Z N)$ is a weak equivalence.

Consider the trivial fibration $c Z(f N) \rightarrow Z(f N)$. Since $\widetilde{T}_{C}(N)$ is cofibrant (since $N$ is), the map $\widetilde{T}_{C}(N) \rightarrow Z(N) \rightarrow Z(f N)$ lifts to a map $\widetilde{T}_{C}(N) \rightarrow c Z(f N)$. This becomes a weak equivalence after applying $\mathbb{P}_{n}$, by the previous paragraph.

In the square

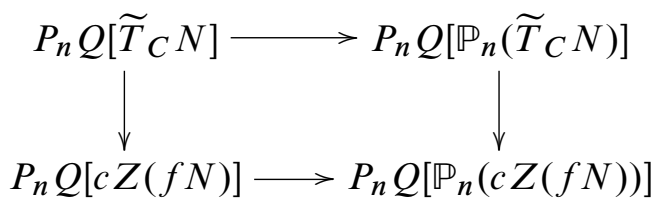

the two horizontal maps are weak equivalences by (6.6). The previous paragraph shows that the right vertical map is a weak equivalence, so the left vertical map is as well. Thus, we have an equivalence

$$
P_{n}(N) \cong P_{n}\left[Q \widetilde{T}_{C}(N)\right] \rightarrow P_{n} Q[c Z(f N)] .
$$

It is easy to use the adjoint functors to see that the composite of this map with our map $g: P_{n} Q[c Z(f N)] \rightarrow P_{n}(f N)$ is the map $P_{n} N \rightarrow P_{n}(f N)$ induced by $N \rightarrow f N$. Since this composite is a weak equivalence, so is the map $g$.

6.7 Proposition Fix $n \geq 1$, and let $N$ be a $C$-bimodule such that $\pi_{i}(N)=0$ for $i \neq n$. If $X \in \mathcal{N}$ on $U_{C}$ is weakly equivalent to $Z(N)$, then the natural map $c X \rightarrow Z P_{n}[Q c X]$ is a weak equivalence.

Proof The natural map in the statement is the composite $\eta: c X \rightarrow Z Q[c X] \rightarrow$ $Z P_{n}[Q c X]$. Since there will necessarily be a weak equivalence $c X \rightarrow Z(f N)$, it suffices to check that $\eta$ is a weak equivalence when $X=Z(f N)$. In this case we consider the diagram

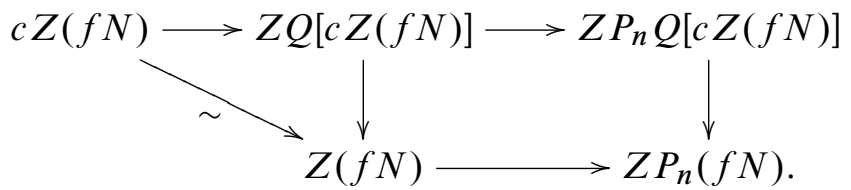

The composite of the top horizontal maps is $\eta$, the vertical maps come from the counit of the $(Q, Z)$ adjunction, and the diagram is readily checked to commute. The bottom horizontal map is a weak equivalence because $f N \rightarrow P_{n}(f N)$ is a weak equivalence, 
and $Z$ preserves all weak equivalences. Finally, we know by the preceding proposition that the right vertical map is a weak equivalence, so $\eta$ is a weak equivalence as well. $\square$

\section{The moduli space of Eilenberg-Mac Lane objects}

Recall where we are at this point in the paper. We have completed the proof of Proposition 4.3, and our next goal is to prove Proposition 4.4.

\subsection{General moduli space technology}

If $\mathcal{C}$ is a model category and $X$ is an object of $\mathcal{C}$, let $\mathcal{M}_{\mathcal{C}}(X)$ denote the category consisting of all objects weakly equivalent to $X$, where the maps are weak equivalences. This is called the Dwyer-Kan classification space of $X$, or the moduli space of $X$. It is a theorem of Dwyer-Kan $[10,2.3]$ that $\left|\mathcal{M}_{\mathcal{C}}(X)\right| \simeq B \operatorname{hAut}(X)$ where $\operatorname{hAut}(X)$ denotes the simplicial monoid of homotopy automorphisms of $X$. This is simply the subcomplex of the homotopy function complex $\underline{\mathrm{e}}(X, X)$ consisting of all path components which are invertible in the monoid $\pi_{0} \underline{\mathrm{e}}(X, X)$.

If $X$ and $Y$ are two objects of $\mathcal{C}$ then we'll write $\mathcal{H}_{\mathrm{C}}(X, Y)$ for the category consisting of diagrams

$$
X \stackrel{\sim}{\longleftarrow} U \longrightarrow V \stackrel{\sim}{\longleftarrow} Y
$$

where the indicated maps are weak equivalences. A morphism in this category is a natural weak equivalence between diagrams which is the identity on $X$ and $Y$. It is another result of Dwyer-Kan that one has a natural zig-zag of weak equivalences between $\mathcal{H}_{\mathrm{Com}}(X, Y)$ and the homotopy function complex $\underline{\mathrm{e}}(X, Y)$. This follows from $[8,6.2(\mathrm{i}), 8.4]$.

When $Y$ is fibrant one may consider a simpler moduli space: let $\mathcal{H}_{\mathrm{C}}(X, Y)^{f}$ be the category whose objects are diagrams

$$
X \stackrel{\sim}{\longleftarrow} U \longrightarrow Y \text {. }
$$

A map in this category is again a weak equivalence of diagrams which is the identity on $X$ and $Y$. There is an inclusion functor $\mathcal{H}_{0} m_{\mathrm{e}}(X, Y)^{f} \rightarrow \mathcal{H}_{\mathrm{C}} \mathrm{m}_{\mathrm{C}}(X, Y)$, and it is stated in $[4,2.7]$ that this is a weak equivalence when $Y$ is fibrant. For a proof, see [5].

7.2 Remark All of the Dwyer-Kan theorems we mentioned above were actually proven only for simplicial model categories. It is not obvious whether the category $R-\mathcal{A l g}$ is simplicial, though. Using the main result of [6], however, the Dwyer-Kan 
results can be immediately extended to all combinatorial model categories. All of the model categories considered in the present paper are combinatorial, so we will freely make use of this technology.

\subsection{Applications to ring spectra}

Note that there is a functor

$$
\Theta: \mathcal{H}_{R-\mathcal{A l g} / C}\left(C, C \vee \Sigma^{n+1} M\right) \rightarrow \mathcal{K}_{C}(M, n)
$$

which sends a diagram

$$
C \stackrel{\sim}{\longleftarrow} U \longrightarrow V \stackrel{\sim}{\longleftarrow} C \vee \Sigma^{n+1} M
$$

in $R-\mathcal{A l g} / C$ to the object of $\mathcal{K}_{C}(M, n)$ represented by

$$
U \rightarrow V \leftarrow C
$$

(where the second map is the composite $C \hookrightarrow C \vee \Sigma^{n+1} M \stackrel{\sim}{\longrightarrow} V$ ).

The following result is very similar to $[4,2.11]$.

7.4 Lemma The sequence of maps

$$
\mathcal{H}^{\circ o m}{ }_{R-\mathcal{A l g} / C}\left(C, C \vee \Sigma^{n+1} M\right) \rightarrow \mathcal{K}_{C}(M, n) \rightarrow \mathcal{M}_{R-\mathcal{A l g} / C}(C) \times \mathcal{E}_{C}(M, n)
$$

is a homotopy fiber sequence of simplicial sets.

Note that $\mathcal{M}_{R-\mathcal{A l g} / C}(C)$ is contractible, as $C$ is a terminal object of this category. So this lemma, together with the identification of $\mathcal{H o m}_{R-\mathcal{A l g}}(X, Y)$ with the homotopy function space $\underline{R-\mathcal{A l g} / C}(X, Y)$, yields Proposition 4.4(a).

Proof The second map is $F: \mathcal{K}_{C}(M, n) \rightarrow \mathcal{M}_{R-\mathcal{A l g} / C}(C) \times \mathcal{E}_{C}(M, n)$ which sends an object $A \rightarrow E \leftarrow B$ to the pair consisting of $A$ and $E \leftarrow B$. The proof of this lemma will be an application of Quillen's Theorem B-however, a little care is required.

Let $\mathcal{K}_{C}^{f}(M, n)$ denote the full subcategory of $\mathcal{K}_{C}(M, n)$ consisting of objects $A \rightarrow$ $E \leftarrow B$ where the maps $E \rightarrow C$ and $B \rightarrow C$ are fibrations. Let $\mathcal{E}_{C}^{f}(M, n)$ denote the analogous subcategory of $\mathcal{E}_{C}(M, n)$. The inclusions $\mathcal{K}_{C}^{f}(M, n) \hookrightarrow \mathcal{K}_{C}(M, n)$ and $\mathcal{E}_{C}^{f}(M, n) \hookrightarrow \mathcal{E}_{C}(M, n)$ are readily checked to be homotopy equivalences. Let

$$
\widetilde{F}: \mathcal{K}_{C}^{f}(M, n) \rightarrow \mathcal{E}_{C}^{f}(M, n)
$$

be the restriction of the functor $F$. 
To apply Quillen's Theorem B [17], we are required to check that for every map $\left[E^{\prime} \leftarrow B^{\prime}\right] \rightarrow\left[E^{\prime \prime} \leftarrow B^{\prime \prime}\right]$ in $\mathcal{E}_{C}^{f}(M, n)$ the induced map of overcategories

$$
\left(\widetilde{F} \downarrow\left[E^{\prime} \leftarrow B^{\prime}\right]\right) \rightarrow\left(\widetilde{F} \downarrow\left[E^{\prime \prime} \rightarrow B^{\prime \prime}\right]\right)
$$

is a weak equivalence. There is a functor

$$
\phi^{\prime}: \mathcal{H}_{\text {om }} \operatorname{Alg}_{/ C}\left(C, E^{\prime}\right)^{f} \rightarrow\left(\widetilde{F} \downarrow\left[E^{\prime} \leftarrow B^{\prime}\right]\right)
$$

sending a diagram $C \stackrel{\sim}{\longleftarrow} \rightarrow E^{\prime}$ to the pair consisting of the object $A \rightarrow E^{\prime} \leftarrow B^{\prime}$ in $\mathcal{K}_{C}^{f}(M, n)$ together with the identity map from $\widetilde{F}\left(A \rightarrow E^{\prime} \leftarrow B^{\prime}\right)$ to $\left[E^{\prime} \leftarrow B^{\prime}\right]$. This functor $\phi^{\prime}$ is readily checked to be a homotopy equivalence. Similarly, one has

$$
\phi^{\prime \prime}: \mathcal{H}_{\operatorname{som}-\mathcal{A l g} / C}\left(C, E^{\prime \prime}\right)^{f} \rightarrow\left(\widetilde{F} \downarrow\left[E^{\prime \prime} \leftarrow B^{\prime \prime}\right]\right)
$$

which is a homotopy equivalence by the same argument. The map

$$
\mathcal{H o m}_{R-\mathcal{A l g} / C}\left(C, E^{\prime}\right)^{f} \rightarrow \mathcal{H} \operatorname{lom}_{R-\mathcal{A l g} / C}\left(C, E^{\prime \prime}\right)^{f}
$$

is a weak equivalence because it is naturally equivalent to the map of function complexes $R-\mathcal{A} \lg _{/ C}\left(C, E^{\prime}\right) \rightarrow R-\mathcal{A l g} / C\left(C, E^{\prime \prime}\right)$ (which is itself a weak equivalence because $\overline{E^{\prime} \rightarrow E^{\prime \prime}}$ is a weak equivalence). So we have established that our map of overcategories is a weak equivalence.

Quillen's Theorem B now tells us that the sequence

$$
\left(\widetilde{F} \downarrow\left[f\left(C \vee \Sigma^{n+1} M\right) \leftarrow C\right]\right) \rightarrow \mathcal{K}_{C}^{f}(M, n) \stackrel{\widetilde{F}}{\longrightarrow} \mathcal{E}_{C}^{f}(M, n)
$$

is a homotopy fiber sequence, where the basepoint in the base space is taken to be the object $\left[f\left(C \vee \Sigma^{n+1} M\right) \leftarrow C\right]$. We have already remarked that the overcategory appearing here is homotopy equivalent to the moduli category

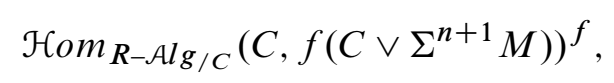

and that the inclusions $\mathcal{K}_{C}^{f}(M, n) \hookrightarrow \mathcal{K}_{C}(M, n)$ and $\mathcal{E}_{C}^{f}(M, n) \hookrightarrow \mathcal{E}_{C}(M, n)$ are homotopy equivalences. So to complete the proof of the lemma it suffices to note two things. First, we have the commutative square

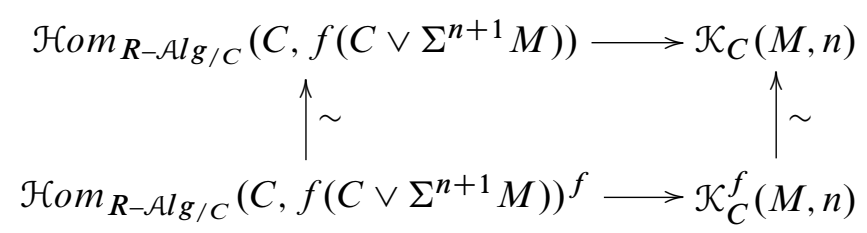


where both vertical maps are the inclusions and the horizontal maps are induced by the functor $\Theta$ defined in Section 7.3. Second, one has a commutative triangle

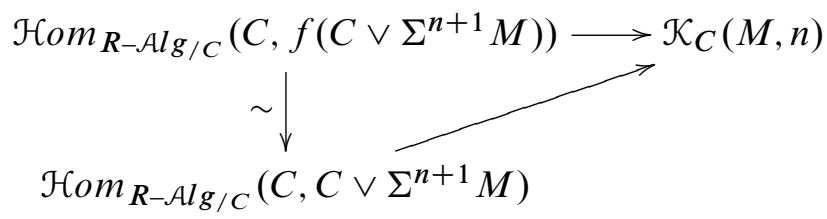

where, again, all the maps are the obvious ones.

Our next goal is to identify $\left|\mathcal{E}_{C}(M, n)\right|$ as in Proposition 4.4(b). We first show that for $C$ a cofibrant $R$-algebra, $\mathcal{E}_{C}(M, n)$ is equivalent to a moduli space in the category $\mathcal{N o n} U_{C}$ of nonunital $C$-algebras. This then reduces further to a moduli space in the category of $C$-bimodules. Computations in the category of $C$-bimodules are relatively simple, so that it is not hard to identify the homotopy type of the moduli space in $C$-bimodules as $B \operatorname{Aut}(M)$. Finally, we remove the cofibrancy condition in Corollary 7.8 .

7.5 Lemma Assume $C$ is a cofibrant $R$-algebra. There are weak equivalences of categories

$$
\mathcal{E}_{C}(M, n) \simeq \mathcal{M}_{(C \downarrow R-\mathcal{A l g \downarrow})}\left(C \vee \Sigma^{n+1} M\right) \simeq \mathcal{M}_{\mathcal{N o n U}_{C}}\left(\Sigma^{n+1} M\right) .
$$

Proof We write $\mathcal{E}$ for $\mathcal{E}_{C}(M, n)$. The argument proceeds in several steps. First, let $\mathcal{E}^{\prime}$ be the full subcategory of $\mathcal{E}$ whose objects $B \rightarrow E$ have $B=C$ (and the map $B \rightarrow C$ the identity). Let $\mathcal{E}^{\prime \prime}$ be the full subcategory of $\mathcal{E}$ whose objects are maps $B \succ E$ in $R-\mathcal{A l g} / C$ in which both $B$ and $E$ are cofibrant $R$-algebras and $B \rightarrow E$ is a cofibration. Finally, let $\mathcal{E}^{\prime \prime \prime}$ be the full subcategory of $\mathcal{E}^{\prime}$ whose objects are in both $\mathcal{E}^{\prime}$ and $\mathcal{E}^{\prime \prime}$. Notice that there is a chain of inclusions

$$
\mathcal{E} \hookleftarrow \mathcal{E}^{\prime \prime} \hookleftarrow \mathcal{E}^{\prime \prime \prime} \hookrightarrow \mathcal{E}^{\prime} .
$$

We claim that each of these inclusions induces a weak equivalence on nerves. This is easy for $\mathcal{E}^{\prime \prime} \hookrightarrow \mathcal{E}$ and $\mathcal{E}^{\prime \prime \prime} \hookrightarrow \mathcal{E}^{\prime}$, just using functorial factorizations.

Define a functor $\theta: \mathcal{E}^{\prime \prime} \rightarrow \mathcal{E}^{\prime \prime \prime}$ by sending an object $B \nrightarrow E$ in $\mathcal{E}^{\prime \prime}$ to the object $C \succ C \amalg_{B} E$. To see that this lies in $\mathcal{E}^{\prime \prime \prime}$ one can use Ken Brown's lemma [12] to show that pushing out a weak equivalence along a cofibration yields another weak equivalence, provided all the domains and codomains of the original maps are cofibrant. It is simple to see that $\theta$ gives a homotopy inverse for the inclusion $\mathcal{E}^{\prime \prime \prime} \hookrightarrow \mathcal{E}^{\prime \prime}$.

If $C \rightarrow X$ lies in $\mathcal{E}^{\prime}$, a straightforward argument shows that $X$ is weakly equivalent to $C \vee \Sigma^{n+1} M$ in the category $(C \downarrow R-\mathcal{A l g} \downarrow C)$ (basically, one uses obstruction theory 
to directly construct a zig-zag of weak equivalences). So $\mathcal{E}^{\prime}$ is simply the moduli space $\mathcal{M}_{(C \downarrow R-\mathcal{A l g \downarrow C )}}\left(C \vee \Sigma^{n+1} M\right)$.

Recall from Proposition 6.2 that there is a Quillen equivalence

$$
\mathcal{N o n} U_{C} \stackrel{\sim}{\longrightarrow}(C \downarrow R-\mathcal{A l g} \downarrow C)
$$

in which the right adjoint $I$ sends $C \rightarrow X \rightarrow C$ to the fiber of $X \rightarrow C$. Note that $I\left(C \vee \Sigma^{n+1} M\right) \simeq \Sigma^{n+1} M$ where $\Sigma^{n+1} M$ is given the trivial structure of nonunital $C$-algebra (in which the product is zero). Thus, this Quillen equivalence implies that $\mathcal{M}_{(C \downarrow R-\mathcal{A l g \downarrow})}\left(C \vee \Sigma^{n+1} M\right) \simeq \mathcal{M}_{\mathcal{N} \text { on } U_{C}}\left(\Sigma^{n+1} M\right)$.

We next reduce from $\mathcal{N o n} U_{C}$ to $C$-bimod:

7.6 Proposition The functor $Z$ induces a weak equivalence

$$
\mathcal{M}_{C-\text { bimod }}\left(\Sigma^{n+1} M\right) \rightarrow \mathcal{M}_{\mathcal{N o n U}_{C}}\left(\Sigma^{n+1} M\right) .
$$

Proof Since $Z$ preserves all weak equivalences, it induces a functor between moduli categories in the obvious way. Consider the composite functor $\mathcal{N}_{\text {on }} U_{C} \rightarrow C$-bimod given by

$$
X \mapsto P_{n+1}(Q(c X)),
$$

where $c$ is any cofibrant-replacement functor in $\mathcal{N}$ on $U_{C}$. Applying Proposition 6.5 with $n$ replaced by $n+1$, we know that if $X$ is weakly equivalent to $Z\left(\Sigma^{n+1} M\right)$ then $P_{n+1}(Q c X)$ is a $C$-bimodule whose only nonvanishing homotopy group lies in dimension $n+1$ and is isomorphic to $M$. So $P_{n+1} Q c$ induces a functor

$$
F: \mathcal{M}_{N_{\text {onU }}}\left(\Sigma^{n+1} M\right) \rightarrow \mathcal{M}_{C-\operatorname{bimod}}\left(\Sigma^{n+1} M\right) .
$$

Proposition 6.5 implies that there is a natural zig-zag of weak equivalences between the composite $F \circ Z$ and the identity functor. Proposition 6.7 implies the same for the composite $Z \circ F$. It follows that the maps induced by $Z$ and $F$ on the nerves of the categories are homotopy inverses.

We now need to analyze $\mathcal{M}_{C \text {-bimod }}\left(\Sigma^{n+1} M\right)$. This is something which boils down to an explicit computation. For the next proposition, recall that to any element $X$ of $\mathcal{M}_{C \text {-bimod }}\left(\Sigma^{n+1} M\right)$ we may associate its homotopy group $\pi_{n+1} X$ regarded as a $\pi_{0}(C)$-bimodule. This bimodule is isomorphic to $M$, and in this way we obtain a functor $\pi_{n+1}: \mathcal{M}_{C \text {-bimod }}\left(\Sigma^{n+1} M\right) \rightarrow \mathcal{M}_{\pi_{0}(C) \text {-bimod }}(M)$. The codomain is the category of $\pi_{0}(C)$-bimodules which are isomorphic to $M$, with maps the isomorphisms; said differently, it is the moduli space in the model category of $\pi_{0}(C)$-bimodules where 
the weak equivalences are isomorphisms and where every map is both a cofibration and a fibration. We use this functor in Corollary 7.8 and Theorem 8.1 to identify the action of $\operatorname{Aut}(M)$.

7.7 Proposition Assume $C$ is cofibrant as an $R$-module (for example, $C$ is a cofibrant $R$-algebra). Then the functor $\pi_{n+1}: \mathcal{M}_{C \text {-bimod }}\left(\Sigma^{n+1} M\right) \rightarrow \mathcal{M}_{\pi_{0}(C) \text {-bimod }}(M)$ is a weak equivalence. Consequently, one has

$$
\mathcal{M}_{C \text {-bimod }}\left(\Sigma^{n+1} M\right) \simeq B \operatorname{Aut}(M)
$$

where the automorphism group is taken in the category of $\pi_{0}(C)$-bimodules.

Proof The Dwyer-Kan result [10, 2.3] identifies $\mathcal{M}_{C \text {-bimod }}\left(\Sigma^{n+1} M\right)$ with the space $B$ hAut $\left(\Sigma^{n+1} M\right)$, where hAut denotes the simplicial monoid of homotopy automorphisms in the model category $C$-bimod. This is the subcomplex of the homotopy function complex $\underline{C-\operatorname{bimod}}\left(\Sigma^{n+1} M, \Sigma^{n+1} M\right)$ consisting of all path components which are invertible in $\pi_{0}$. We'll now compute this homotopy function complex.

The model category of $C$-bimodules is enriched, tensored, and cotensored over symmetric spectra. So for any bimodules $N_{1}$ and $N_{2}$ there is a symmetric spectrum mapping space $F\left(N_{1}, N_{2}\right)$. The homotopy function complex is simply the zero-th space $\operatorname{Ev}_{0} F\left(c N_{1}, f N_{2}\right)$. Also, since $C$-bimod is a stable model category one has $F\left(\Sigma c N_{1}, f \Sigma c N_{2}\right) \simeq F\left(c N_{1}, f N_{2}\right)$. We need to use $F\left(c \Sigma^{n+1} M, f \Sigma^{n+1} M\right) \simeq$ $F(c f M, c f M)$.

It is simple to compute that $\pi_{i} F(c f M, c f M)=0$ for $i>0$ and $\pi_{0} F(c f M, c f M)=$ $\operatorname{Hom}_{\pi_{0}(C)}(M, M)$, the group of endomorphisms of $M$ as a $\pi_{0}(C)$-bimodule. One way to do this is to recall that for any two $C$-bimodules $N_{1}$ and $N_{2}$ there is a spectral sequence

$$
E_{2}^{p, q}=\operatorname{Ext}_{\pi_{*}\left(C \wedge_{R} C^{o p}\right)}^{p}\left(\pi_{*}\left(N_{1}\right), \Sigma^{-q} \pi_{*}\left(N_{2}\right)\right) \Rightarrow \pi_{q-p} F\left(c N_{1}, f N_{2}\right) .
$$

In the case $N_{1}=N_{2}=M$ the $E_{2}$-term completely vanishes in the range $q \geq 0$ except for the single group $\operatorname{Ext}_{\pi_{*}\left(C \wedge_{R} C^{o p}\right)}^{0}(M, M)$ when $p=q=0$ (this uses that $C \wedge_{R} C$ is connective, which in turn uses our cofibrancy assumption on $C$ ). This group is the same as $\operatorname{Hom}_{\pi_{0}}\left(C \wedge_{R} C^{o p}\right)(M, M)$. Finally, we note that $\pi_{0}\left(C \wedge_{R} C^{o p}\right) \cong$ $\pi_{0}(C) \otimes_{\pi_{0} R} \pi_{0}(C)^{o p}$. This follows from the spectral sequence

$$
\operatorname{Tor}_{p, q}^{\pi_{*} R}\left(\pi_{*} C, \pi_{*} C^{o p}\right) \Rightarrow \pi_{p+q}\left(C \wedge_{R} C^{o p}\right)
$$

(again using our cofibrancy assumption on $C$ ), together with the fact that $R$ and $C$ are connective. 
Putting this all together, it readily follows that $\operatorname{hAut}\left(\Sigma^{n+1} M\right) \simeq \operatorname{Aut}(M)$ (the latter regarded as a discrete group).

Finally, we return to $\pi_{n+1}: \mathcal{M}_{C \text {-bimod }}\left(\Sigma^{n+1} M\right) \rightarrow \mathcal{M}_{\pi_{0}(C) \text {-bimod }}(M)$. Since the homotopy groups of both the domain and codomain vanish except for $\pi_{1}$, it suffices to show the functor induces an isomorphism on $\pi_{1}$. Note that there are obvious maps

$$
\operatorname{Aut}(M) \rightarrow \pi_{1} \mathcal{M}_{C-\operatorname{bimod}}\left(\Sigma^{n+1} M\right) \quad \text { and } \quad \operatorname{Aut}(M) \rightarrow \pi_{1} \mathcal{M}_{\pi_{0}(C) \text {-bimod }}(M) .
$$

The first, for instance, sends an automorphism $\sigma$ to the loop represented by the induced map of bimodules $\sigma: \Sigma^{n+1} M \rightarrow \Sigma^{n+1} M$; the second is defined similarly. These maps obviously commute with the functor $\pi_{n+1}$. But the map $\operatorname{Aut}(M) \rightarrow$ $\pi_{1} \mathcal{M}_{\pi_{0}(C) \text {-bimod }}(M)$ is readily seen to be an isomorphism, and our analysis above of $\operatorname{hAut}\left(\Sigma^{n+1} M\right)$ shows that the corresponding map $\operatorname{Aut}(M) \rightarrow \pi_{1} \mathcal{M}_{C \text {-bimod }}\left(\Sigma^{n+1} M\right)$ is also an isomorphism. This finishes the proof.

To any object $E \leftarrow B$ in $\mathcal{E}_{C}(M, n)$ we may associate the abelian group $\pi_{n+1} E$ which will be a $\pi_{0}(C)$-bimodule via the isomorphism $\pi_{0}(B) \cong \pi_{0}(C)$ and the map $\pi_{0}(B) \rightarrow \pi_{0}(E)$. So we have a functor $\pi_{n+1}: \mathcal{E}_{C}(M, n) \rightarrow \mathcal{M}_{\pi_{0}(C) \text {-bimod }}(M)$.

7.8 Corollary The functor $\pi_{n+1}: \mathcal{E}_{C}(M, n) \rightarrow \mathcal{M}_{\pi_{0}(C) \text {-bimod }}(M)$ is a weak equivalence. Consequently, $\varepsilon_{C}(M, n) \simeq B \operatorname{Aut}(M)$.

Proof Let $c C \stackrel{\sim}{\longrightarrow} C$ be a cofibrant-replacement in the category of $R$-algebras. By Proposition 4.2 the evident map $\varepsilon_{c C}(M, n) \rightarrow \mathcal{E}_{C}(M, n)$ is a weak equivalence. We then have a zig-zag of weak equivalences

$$
\mathcal{E}_{C}(M, n) \simeq \mathcal{E}_{c C}(M, n) \simeq \mathcal{M}_{N o n U_{c C}}\left(\Sigma^{n+1} M\right) \simeq \mathcal{M}_{c C-\operatorname{bimod}}\left(\Sigma^{n+1} M\right)
$$

provided by Lemma 7.5 and Proposition 7.6. There is an obvious $\pi_{n+1}$ functor from each of these categories landing in $\mathcal{M}_{\pi_{0}(C) \text {-bimod }}(M)$, and the relevant triangles all commute. Since $\pi_{n+1}: \mathcal{M}_{c} C$-bimod $\left(\Sigma^{n+1} M\right) \rightarrow \mathcal{M}_{\pi_{0}(C) \text {-bimod }}(M)$ is a weak equivalence by Proposition 7.7, we deduce that the same is true for the $\pi_{n+1}$ functor with domain $\mathcal{E}_{C}(M, n)$.

We have finally completed our main proof:

Proof of Proposition 4.4 Part (a) follows directly from Lemma 7.4 and the remarks following its proof. Part (b) is a consequence of the preceding corollary. 


\section{The main result}

Finally, we can pull everything together and prove the main theorem:

8.1 Theorem Fix $n \geq 1$. Let $R$ be a connective ring spectrum, and let $C$ be a connective $R$-algebra such that $P_{n-1} C \simeq C$. Let $M$ be a $\pi_{0}(C)$-bimodule. There is a homotopy fiber sequence of spaces

$$
\underline{R-\mathcal{A} \lg _{/ C}}\left(C, C \vee \Sigma^{n+1} M\right) \rightarrow|\mathcal{M}(C+(M, n))| \rightarrow B \operatorname{Aut}(M) .
$$

Consequently, one has a bijection

$$
\left[H o(R-\mathcal{A l g} / C)\left(C, C \vee \Sigma^{n+1} M\right)\right] / \operatorname{Aut}(M) \cong \pi_{0} \mathcal{M}(C+(M, n))
$$

where $\operatorname{Aut}(M)$ acts on the second factor of $C \vee \Sigma^{n+1} M$.

Proof First one uses that $\mathcal{M}(C+(M, n)) \simeq \mathcal{K}_{C}(M, n)$, from Proposition 4.3. Then one uses the homotopy fiber sequence

$$
\mathcal{M}_{R-\mathcal{A l g} / C}\left(C, C \vee \Sigma^{n+1} M\right) \rightarrow \mathcal{K}_{C}(M, n) \rightarrow \mathcal{E}_{C}(M, n)
$$

established in Lemma 7.4 and our identification $\mathcal{E}_{C}(M, n) \simeq B \operatorname{Aut}(M)$. This proves the first claim of the theorem.

To prove the second statement, we look at the long exact homotopy sequence for the above fiber sequence. Since $\mathcal{E}_{C}(M, n)$ is connected, this identifies $\pi_{0} \mathcal{K}_{C}(M, n)$ with a quotient of $\pi_{0}\left[\mathcal{M}_{R-\mathcal{A l g} / C}\left(C, C \vee \Sigma^{n+1} M\right)\right]$ by an action of $\pi_{1} \varepsilon_{C}(M, n) \cong \operatorname{Aut}(M)$. We must identify the action.

For brevity, write $S=\pi_{0}\left[\mathcal{M}_{R-\mathcal{A l g} / C}\left(C, C \vee \Sigma^{n+1} M\right)\right]$. Every equivalence class $s \in S$ can be represented by a diagram

$$
C \stackrel{\sim}{\longleftarrow} A \stackrel{g}{\longrightarrow} f\left(C \vee \Sigma^{n+1} M\right) \longleftarrow C \vee \Sigma^{n+1} M .
$$

Let $\sigma \in \operatorname{Aut}(M)$. Under the identification $\pi_{1} \varepsilon_{C}(M, n) \cong \operatorname{Aut}(M), \sigma$ corresponds to the self-map of the object $\left[C \hookrightarrow C \vee \Sigma^{n+1} M\right]$ which is the identity on $C$ and induced by $\sigma$ on $M$. We can just as well represent $\sigma$ as a self-map of $\left[C \hookrightarrow f\left(C \vee \Sigma^{n+1} M\right)\right]$.

To determine the action of $\sigma$ on $s$ we do the usual thing: we lift the loop represented by $\sigma$ to a path in $\mathcal{K}_{C}(M, n)$ beginning at $s$, and we take the terminal point of that path. Our path is the map

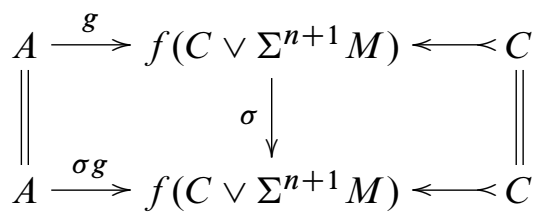

Algebraic 83 Geometric Topology, Volume 6 (2006) 
This identifies the action of $\operatorname{Aut}(M)$ on $S=\operatorname{Ho}(R-\mathcal{A l g} / C)\left(C, C \vee \Sigma^{n+1} M\right)$ with the action coming from the second factor of $C \vee \Sigma^{n+1} M$.

8.2 Remark The isomorphism from $\left[\operatorname{Ho}(R-\mathcal{A l g} / C)\left(C, C \vee \Sigma^{n+1} M\right)\right] / \operatorname{Aut}(M)$ to $\pi_{0} \mathcal{M}(C+(M, n))$ produced in the theorem is precisely the pullback map $\widetilde{\mathrm{PB}}$ defined in Section 3.6. This follows at once by looking at the various maps we used in our identifications (particularly the one of Proposition 4.3).

8.3 Remark Theorem 1.3 and Corollary 1.4 from the introduction are the special case of the above theorem where $R=S$.

8.4 Remark In [4] the moduli problem for Postnikov extensions of spaces is set up a bit differently. Proposition 3.7 in [4] considers all spaces $Y \simeq P_{n} Y$ for which there exists some chain of weak equivalences $P_{n-1} Y \simeq C$, instead of considering maps $Y \rightarrow C$ with a fixed space $C$ as the $(n-1)$ st Postnikov section. (Note that with the definition of [4] one must be careful about the isomorphism $\pi_{n+1} Y \cong M$, as this must be an isomorphism of bimodules and to make sense of this one needs a fixed isomorphism of rings $\pi_{0} Y \cong \pi_{0} C$ ).

If one adopts the choices of [4], one obtains a homotopy fiber sequence analogous to Theorem 8.1 where the base space is a product $\mathcal{M}(C) \times B \operatorname{Aut}(M)$ and $\mathcal{M}(C)$ is the moduli space of $R$-algebras weakly equivalent to $C$. See $[4,3.10]$. It is probably possible to directly relate our Theorem 8.1 to this formulation, although we have not pursued this.

\subsection{Alternative formulations and the connection with THH}

Let $R, C, M$, and $n$ be as in the statement of Theorem 8.1. In a typical application, one is interested in the possible extensions of $C$ by $M$ and thus is led to try to compute Ho $\left(R-\mathcal{A} l g_{/ C}\right)\left(C, C \vee \Sigma^{n+1} M\right)$. There are two well-known ways to simplify this, which we briefly record here.

In some applications one is interested in the following reduction. We don't use it in the present paper, but it makes sense to record it here.

8.6 Proposition Assume $C$ is a cofibrant $R$-algebra and fix a zero-th Postnikov section $p_{0}: C \rightarrow P_{0} C$. One has a weak equivalence of homotopy function complexes

$$
\underline{R-\mathcal{A l g} / C}\left(C, C \vee \Sigma^{n+1} M\right) \simeq \underline{R-\mathcal{A l g} / P_{0} C}\left(C, P_{0} C \vee \Sigma^{n+1} M\right) .
$$


Proof First note that one has a Quillen pair $L: R-\mathcal{A l g} / C \rightleftarrows R-\mathcal{A l g} / P_{0} C: T$ where $L$ is composition with $p_{0}$ and $T$ is base-change along $p_{0}$. This induces a weak equivalence of homotopy function complexes

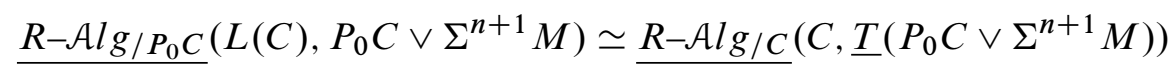

where $T$ denotes the derived functor of $T$ (and where we have used that $C$ is cofibrant). Thus, it suffices for us to show that $\underline{T}\left(P_{0} C \vee \Sigma^{n+1} M\right) \simeq C \vee \Sigma^{n+1} M$ in $R-\mathcal{A l g} / C$.

Factor the projection $P_{0} C \vee \Sigma^{n+1} M \rightarrow P_{0} C$ into a trivial cofibration followed by a fibration $P_{0} C \vee \Sigma^{n+1} M \stackrel{i}{\rightarrow} f P C \stackrel{h}{\rightarrow} P_{0} C$. Define $f C$ as the pullback in the following square

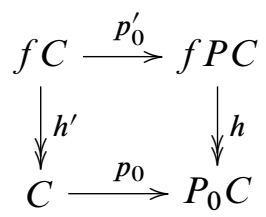

and note that $f C$ is a model for $\underline{T}\left(P_{0} C \vee \Sigma^{n+1} M\right)$.

Observe that the map $C \vee \Sigma^{n+1} M \rightarrow P_{0} C \vee \Sigma^{n+1} M \rightarrow f P C$ factors through $f C$. To see that $C \vee \Sigma^{n+1} M \rightarrow f C$ is a weak equivalence note that in the category of spectra the homotopy fiber of both $f C \rightarrow C$ and $C \vee \Sigma^{n+1} M \rightarrow C$ is $\Sigma^{n+1} M$. $\square$

The second well-known simplification is given by a connection with topological Hochschild cohomology, THH* ${ }^{*}$ This is via three Quillen pairs with the left adjoints on top:

$$
R-\mathcal{A l g} / C \underset{G}{\stackrel{F}{\rightleftarrows}}(C \downarrow R-\mathcal{A l g} \downarrow C) \underset{I}{\stackrel{K}{\rightleftarrows}} \mathcal{N o n U} C \underset{Z}{\stackrel{Q}{\rightleftarrows}} C \text {-bimod }
$$

The second two of these were defined in Section 6. In the first, we have $F(X)=C \amalg_{R} X$ and $G$ is the forgetful functor. Recall that the $(K, I)$ pair is a Quillen equivalence by Proposition 6.2.

Following [1] (but using slightly different notation), for $X \in R-\mathcal{A} l g_{/ C}$ one defines

$$
\Omega_{R \rightarrow C}(X)=(\underline{Q} \circ \underline{I} \circ \underline{F})(X) .
$$

Recall that the underlines denote derived functors. One obtains a chain of weak equivalences

$$
\begin{aligned}
& \underline{R-\mathcal{A l g} / C}\left(C, C \vee \Sigma^{n+1} M\right) \simeq \underline{(C \downarrow R-\mathcal{A} l g \downarrow C)}\left(\underline{F}(C), C \vee \Sigma^{n+1} M\right) \\
& \simeq \underline{\mathcal{N o n U}_{C}}\left(\underline{I}(\underline{F C}), \Sigma^{n+1} M\right) \\
& \simeq \underline{C-\operatorname{bimod}}\left(\Omega_{R \rightarrow C}(C), \Sigma^{n+1} M\right) \text {. }
\end{aligned}
$$


In this chain we have used three facts, namely

$$
\underline{G}\left(C \vee \Sigma^{n+1} M\right) \simeq C \vee \Sigma^{n+1} M, \quad \underline{I}\left(C \vee \Sigma^{n+1} M\right) \simeq \Sigma^{n+1} M,
$$

and

$$
\underline{Z}\left(\Sigma^{n+1} M\right) \simeq \Sigma^{n+1} M .
$$

The first and last are trivial. For the second, use the fact that the evident map from $\Sigma^{n+1} M$ to the homotopy fiber of $C \vee \Sigma^{n+1} M \rightarrow C$ is a weak equivalence.

Assume that $C$ is cofibrant as an $R$-module, and recall that $C$-bimod denotes the category of $\left(C \wedge{ }_{R} C^{o p}\right)$-modules. One defines the group of derivations

$$
\begin{aligned}
\operatorname{Der}_{R}^{n+1}(C, M) & =\operatorname{Ho}(C-\text { bimod })\left(\Omega_{R \rightarrow C}(C), \Sigma^{n+1} M\right) \\
& =\pi_{-n-1}\left[\underline{C-\operatorname{bimod}}\left(\Omega_{R \rightarrow C}(C), M\right)\right] .
\end{aligned}
$$

Note that this is just $\pi_{0}$ of $R-\mathcal{A l g} / C\left(C, C \vee \Sigma^{n+1} M\right)$ by the weak equivalence of mapping spaces given above.

One also defines

$$
\mathrm{THH}_{R}^{k}(C, M)=\operatorname{Ho}(C-\operatorname{bimod})\left(C, \Sigma^{k} M\right)=\pi_{-k}[\underline{C-\operatorname{bimod}}(C, M)] .
$$

To connect these groups, one identifies $\Omega_{R \rightarrow C}(C)$ with the homotopy fiber of the multiplication map

$$
C \wedge_{R} C^{o p} \rightarrow C
$$

(this uses that $C$ is cofibrant as an $R$-module, otherwise a cofibrant-replacement is necessary before forming the smash product). This identification is nontrivial, but a proof has been shown to us by Mike Mandell [15].

8.7 Remark In [14] the bimodule $\Omega_{R \rightarrow C}(C)$ is defined to be the homotopy fiber of the above multiplication map. The hard work is then to prove that the mapping spaces $\underline{C-\operatorname{bimod}}\left(\Omega_{R \rightarrow C}(C), \Sigma^{n+1} M\right)$ and $\underline{R-\mathcal{A l g} / C}\left(C, C \vee \Sigma^{n+1} M\right)$ are weakly equivalent. The proof of this fact in [14] contains gaps.

Applying $\underline{C \text {-bimod }}\left(-, \Sigma^{n+1} M\right)$ to the homotopy fiber sequence $\Omega_{R \rightarrow C}(C) \rightarrow$ $C \wedge_{R} C \rightarrow C$ induces a homotopy fiber sequence of mapping spaces. Consider the associated long exact homotopy sequence. One has $\pi_{i}\left(C-\operatorname{bimod}\left(C \wedge_{R} C, \Sigma^{n+1} M\right)\right) \cong$ $\pi_{i}\left(\Sigma^{n+1} M\right)$; so as long as $n \geq 0$, this group vanishes for $i \leq 0$. The long exact sequence then shows that for $n \geq 0$,

$$
\operatorname{Der}_{R}^{n+1}(C, M) \cong \mathrm{THH}_{R}^{n+2}(C, M) .
$$

Putting everything together, we have proven the following: 
8.8 Proposition Let $R, C, M$, and $n$ be as in Theorem 8.1. Assuming that $C$ is cofibrant as an $R$-module, one has a bijection

$$
\operatorname{THH}_{R}^{n+2}(C, M) / \operatorname{Aut}(M) \cong \pi_{0} \mathcal{M}(C+(M, n)) .
$$

8.9 Remark In the case where $C$ is not cofibrant as an $R$-module, one can repeat the above discussion by replacing $C$-bimod with $Q C$-bimod, where $Q C \rightarrow C$ is a cofibrant-replacement for $C$ in $R-\mathcal{A l g}$. The correct definition of $\mathrm{THH}_{R}^{i}(C, M)$ should really be Ho $\left(Q C\right.$-bimod) $\left(Q C, \Sigma^{i} M\right)$, and analogously for Der.

\section{Appendix A Proof of the Blakers-Massey theorem for ring spectra}

In this section we will give the proof of Theorem 2.3. To ease notation, refer to a map $f: X \rightarrow Y$ as an $(n-1)$-equivalence if $\pi_{i}(Y, X)=0$ for $i<n$. Recall our conventions that we replace $f$ by a cofibration before considering relative homotopy, and that homotopy groups always refer to the derived homotopy groups ( $\pi_{*}$ applied to a fibrant replacement).

We actually prove the following result. For this statement let $\amalg$ denote the coproduct of $R$-algebras and $\cup$ denote the coproduct of $R$-modules. For example, in this notation the homotopy pushout appearing in Theorem 2.3 is $P=C \amalg_{A}^{h} B$.

A.1 Theorem Let $R$ be a connective, commutative ring spectrum and let $m, n \geq 1$. Suppose given two maps of $R$-algebras: $A \rightarrow B$ an $(n-1)$-equivalence and $A \rightarrow C$ an $(m-1)$-equivalence, with $A$ connective. Then the map from the homotopy pushout of $R$-modules to the homotopy pushout of $R$-algebras, $C \cup_{A}^{h} B \rightarrow C \amalg_{A}^{h} B$, is an $(m+n-1)$-equivalence.

We prove this proposition at the end of this section. To get an idea of why it should be true, it's useful to think about the analogous result for dgas. There, by replacing $B$ up to quasi-isomorphism one can assume that $A \rightarrow B$ is a monomorphism which is the identity in degrees strictly smaller than $n$. Similarly, one can assume $A \rightarrow C$ is a monomorphism which is the identity in degrees strictly smaller than $m$. The coproduct $B \amalg_{A} C$ is constructed from formal words in the elements of $B$ and $C$, and inspection shows immediately that $B \cup_{A} C \rightarrow B \amalg_{A} C$ is an isomorphism in degrees less than $m+n$. Our proof for the ring spectra case will have a similar flavor, but it must contend with the fact that ring spectra do not have "elements". 
A.2 Remark Throughout this section we need to deal with colimits both of $R-$ modules and $R$-algebras. There are two basic facts which will be used repeatedly. First, colimits in the category of $R$-modules are the same as colimits in the category of $S$-modules (ie in the category of spectra). Said better, the underlying spectrum of a colimit of $R$-modules is the same as the colimit of the underlying spectra.

The second fact we will need is that for a sequential colimit of $R$-algebras, the colimit in the category of $R$-algebras is the same as the colimit in the category of $R$-modules. Both of these facts work quite generally in the context of arbitrary symmetric monoidal categories.

Before tackling the proof of Theorem A.1, we show how the result implies the BlakersMassey theorem:

Proof of Theorem 2.3 We can assume that $A, B$ and $C$ are cofibrant and fibrant as $R$-algebras (and hence fibrant as spectra). We can also assume $A \rightarrow B$ and $A \rightarrow C$ are cofibrations of $R$-algebras and hence also cofibrations of $R$-modules by [18, 4.1(3)]. Then the homotopy pushout of $R$-modules is the pushout $C \cup_{A} B$ and the homotopy pushout of $R$-algebras is the pushout $P=C \amalg_{A} B$.

By Theorem A.1, we know that $C \cup_{A} B \rightarrow C \amalg_{A} B$ is an $(m+n-1)$-equivalence. It follows that $\pi_{i}\left(C \cup_{A} B, C\right) \rightarrow \pi_{i}(P, C)$ is an isomorphism for $i<m+n-1$ and a surjection for $i=m+n-1$. But $\pi_{i}\left(C \cup_{A} B, C\right) \cong \pi_{i}\left(\left(C \cup_{A} B\right) / C\right) \cong \pi_{i}(B / A) \cong$ $\pi_{i}(B, A)$. So $\pi_{i}(B, A) \rightarrow \pi_{i}(P, C)$ is an isomorphism for $i<m+n-1$ and a surjection for $i=m+n-1$.

The proof of Theorem A.1 will require several lemmas. Although the next lemma is stated for an arbitrary $(n-1)$-connected cofibration $K \succ L$ of pointed simplicial sets, the main application is when $K \rightarrow L$ is $\partial \Delta^{n} \rightarrow \Delta^{n}$ or a coproduct of such maps. Let $\pi_{i}^{s}(L, K)$ denote $\pi_{i}\left(\Sigma^{\infty} L, \Sigma^{\infty} K\right)$. Recall that for a pointed simplicial set $K$, $T_{R}(K)$ is our shorthand for the tensor algebra $T_{R}\left(R \wedge \Sigma^{\infty} K\right)$.

A.3 Lemma Let $n \geq 2$ and let $R$ be a connective, commutative ring spectrum. Let $K \succ L$ be a cofibration of pointed simplicial sets such that $\pi_{i}^{s}(L, K)=0$ for $i<n$. Suppose $X$ is a cofibrant, connective $R$-algebra and

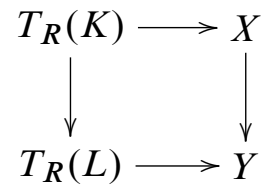

is a pushout square of $R$-algebras. Then $X \rightarrow Y$ is an $(n-1)$-equivalence. 
Proof In [18, Proof of Lemma 6.2] the pushout $Y$ is described as a certain directed colimit of pushouts in the category of $R$-modules. If we let $P_{0}=X$, then there is a sequence of cofibrations of $R$-modules

$$
P_{0} \succ P_{1} \succ P_{2} \succ \cdots
$$

whose colimit is the underlying $R$-module of $Y$ and where $P_{r}$ is obtained from $P_{r-1}$ by a pushout diagram of $R$-modules

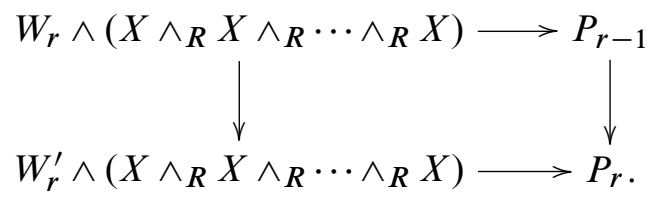

Here there are $r+1$ copies of $X$ in the smash product, and $W_{r} \rightarrow W_{r}^{\prime}$ is the $r$-fold box product of $K \rightarrow L$. So $W_{r}^{\prime} / W_{r} \cong(L / K)^{\wedge r}$, which is $(r n-1)$-connected.

Note that $X \wedge_{R} X \wedge_{R} \cdots \wedge_{R} X$ is connective, since both $X$ and $R$ are connective and $X$ is cofibrant. This follows from the fact that $X \wedge_{R} X$ is the realization of the simplicial $R$-module $[n] \mapsto X \wedge R^{\wedge n} \wedge X$, for instance. So we find that the $R$-module

$$
P_{r} / P_{r-1} \cong(L / K)^{\wedge r} \wedge\left(X \wedge_{R} \wedge \cdots \wedge_{R} X\right)
$$

has no homotopy groups below dimension $r n$. Hence, $P_{r-1} \rightarrow P_{r}$ is an $(r n-1)-$ equivalence. It follows immediately that $X \rightarrow Y$ is an $(n-1)$-equivalence.

Consider the following subset of the generating cofibrations of $R$-algebras: $I_{n}^{\prime}=$ $\left\{T_{R}\left(\partial \Delta^{l}\right) \rightarrow T_{R}\left(\Delta^{l}\right) \mid l \geq n\right\}$. Let $I_{n}=I_{n}^{\prime} \cup J$ where $J$ is the set of generating trivial cofibrations of $R$-algebras. Note that each of these maps is an $(n-1)$-equivalence. The next lemma converts any $(n-1)$-equivalence of $R$-algebras into a weakly equivalent map built from $I_{n}$ by colimits and pushouts. Let $I_{n}$-Cell be the collection of maps which are (possibly infinite) compositions of pushouts of maps in $I_{n}[12,2.1 .9]$.

A.4 Lemma If $f: A \rightarrow B$ is an $(n-1)$-equivalence between fibrant $R$-algebras, then there is a factorization $A \stackrel{i}{\rightarrow} B^{\prime} \stackrel{p}{\rightarrow} B$ with $f=p i, i \in I_{n}$-Cell and $p$ a trivial fibration.

Proof The small object argument produces a factorization of $f$ as $f=p i$ such that $i: A \rightarrow B^{\prime}$ is in $I_{n}$-Cell and $p: B^{\prime} \rightarrow B$ has the right lifting property with respect to $I_{n}$. Since the maps in $J$ are trivial cofibrations, any map in $J$-Cell is a weak equivalence. Thus, by Lemma A.3, $i$ is a (possibly infinite) composition of $(n-1)$-equivalences. So $i$ is an $(n-1)$-equivalence. It follows that $p$ is an $(n-1)$-equivalence as well; hence $\pi_{i}\left(B, B^{\prime}\right)=0$ for $i<n$. The map $p$ is a fibration since it has the right lifting 
property with respect to $J$; hence, $B$ and $B^{\prime}$ are both fibrant. Using the definition in Section 2.4 of relative homotopy groups, it follows that $\pi_{i}\left(B, B^{\prime}\right)=0$ for $i \geq n$ as well since $p$ has the right lifting property with respect to $I_{n}^{\prime}$. Thus, $p$ is a trivial fibration.

A.5 Lemma Assume $R, K$ and $L$ satisfy the hypotheses of Lemma A.3. Suppose $X$ and $C$ are cofibrant, connective $R$-algebras, $X \rightarrow C$ is an $(m-1)$-equivalence and the following two squares

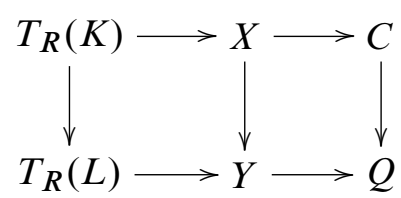

are both pushout squares of $R$-algebras. Then the map from the coproduct in $R-$ modules to the coproduct of $R$-algebras, $C \cup_{X} Y \rightarrow C \sqcup_{X} Y=Q$, is an $(m+n-1)$ equivalence.

Note here $Y=X \amalg_{T_{R}(K)} T_{R}(L)$ and $Q=C \amalg_{X} Y=C \amalg_{T_{R}(K)} T_{R}(L)$.

Proof As described in the proof of Lemma A.3, $Y$ is the colimit of a sequence of cofibrations of $R$-modules

$$
X=P_{0} \succ P_{1} \succ P_{2} \succ \cdots
$$

with $P_{r} / P_{r-1} \cong(L / K)^{\wedge r} \wedge\left(X \wedge_{R} \cdots \wedge_{R} X\right)$. Similarly, one can produce $Q$ as a colimit of a sequence of cofibrations of $R$-modules

$$
C=Q_{0} \succ Q_{1} \succ Q_{2} \succ \cdots
$$

with $Q_{r} / Q_{r-1} \cong(L / K)^{\wedge r} \wedge\left(C \wedge{ }_{R} \cdots \wedge{ }_{R} C\right)$. The map $X \rightarrow C$ induces a map of sequences $P_{i} \rightarrow Q_{i}$.

Next we build a sequence $P_{i}^{\prime}$ between $P_{i}$ and $Q_{i}$. Set $P_{0}^{\prime}=C$ and define $P_{i}^{\prime}$ as the pushout of $R$-modules $P_{i-1}^{\prime} \cup_{P_{i-1}} P_{i}$; so each square in the following diagram is a pushout of $R$-modules.

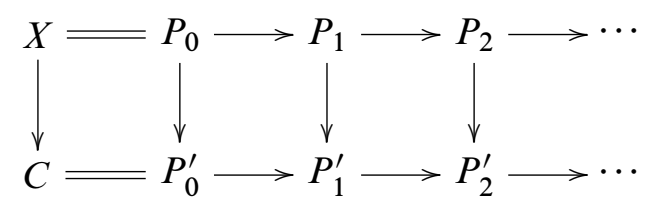

Since colim $P_{i}=Y$, it follows formally that colim $P_{i}^{\prime}=C \cup_{X} Y$. Also note that the maps $P_{i} \rightarrow Q_{i}$ induce maps $f_{i}: P_{i}^{\prime} \rightarrow Q_{i}$. 
We next show inductively that each $f_{i}: P_{i}^{\prime} \rightarrow Q_{i}$ is an $(m+n-1)$-equivalence. First, $f_{0}: C \rightarrow C$ is the identity, and hence an $(m+n-1)$ equivalence. By construction one has $P_{r}^{\prime} / P_{r-1}^{\prime} \cong P_{r} / P_{r-1} \cong(L / K)^{\wedge r} \wedge\left(X \wedge_{R} \cdots \wedge_{R} X\right)$ and $Q_{r} / Q_{r-1} \cong$ $(L / K)^{\wedge r} \wedge\left(C \wedge_{R} \cdots \wedge_{R} C\right)$. Note that $X \wedge_{R} \cdots \wedge_{R} X \rightarrow C \wedge_{R} \cdots \wedge_{R} C$ is the composite

$$
X^{\wedge_{R}(r+1)} \rightarrow C \wedge_{R}\left(X^{\wedge_{R}(r)}\right) \rightarrow C \wedge_{R} C \wedge_{R}\left(X^{\wedge_{R}(r-1)}\right) \rightarrow \cdots \rightarrow C^{\wedge_{R}(r+1)} .
$$

Each map in this sequence comes from smashing $X \rightarrow C$ over $R$ with a connected (and cofibrant) $R$-module, and so each map is an $(m-1)$-equivalence. The spectrum $(L / K)^{\wedge r}$ is $(r n-1)$-connected, so

$$
(L / K)^{\wedge r} \wedge\left(X^{\wedge R}(r+1)\right) \rightarrow(L / K)^{\wedge r} \wedge\left(C^{\wedge R}(r+1)\right)
$$

is an $(r n+m-1)$-equivalence. Hence $P_{r}^{\prime} / P_{r-1}^{\prime} \rightarrow Q_{r} / Q_{r-1}$ is, in particular, an $(m+n-1)$-equivalence.

Since $P_{r-1}^{\prime} \rightarrow Q_{r-1}$ is an $(m+n-1)$-equivalence by induction, it now follows that $P_{r}^{\prime} \rightarrow Q_{r}$ is also an $(m+n-1)$-equivalence. So this holds for all $r$. Since homotopy groups commute with colimits of $R$-modules, this implies that colim $P_{r}^{\prime} \rightarrow \operatorname{colim} Q_{r}$ is an $(m+n-1)$-equivalence.

Finally we can complete the main result of this section:

Proof of Theorem A.1 We can assume that $A, B$, and $C$ are cofibrant and fibrant as $R$-algebras (hence fibrant as spectra). By Lemma A.4, we can assume $A \rightarrow B$ is in $I_{n}$-Cell. We are now in a situation where the homotopy pushouts are weakly equivalent to the pushouts, so our task is to show that $C \cup_{A} B \rightarrow C \amalg_{A} B$ is an $(m+n-1)$-equivalence.

Since $A \rightarrow B$ is in $I_{n}$-Cell we can assume $B=$ colim $B_{i}$ with $B_{0}=A$ and each $B_{i-1} \rightarrow B_{i}$ a pushout of a coproduct of maps in $I_{n}=I_{n}^{\prime} \cup J$. Any coproduct of maps in $I_{n}^{\prime}$ has the form $T_{R}(K) \rightarrow T_{R}(L)$ for some cofibration of pointed simplicial sets $K \rightarrow L$ which is a stable $(n-1)$-equivalence. And any coproduct of maps in $J$ is a trivial cofibration. So any coproduct of maps in $I_{n}$ has the form

$$
T_{R}(K) \amalg W^{\prime} \rightarrow T_{R}(L) \amalg Z^{\prime}
$$

where $W^{\prime} \rightarrow Z^{\prime}$ is a trivial cofibration. Forming $B_{i}$ from $B_{i-1}$ can therefore be done in two stages, by first pushing out along the map $T_{R}(K) \rightarrow T_{R}(L)$ and then pushing out along the map $W^{\prime} \rightarrow Z^{\prime}$. By now redefining the $B_{i}$ 's, we can assume that each map $B_{i-1} \rightarrow B_{i}$ in our colimit is obtained by pushing out either along a trivial cofibration or along a map $T_{R}(K) \rightarrow T_{R}(L)$ as above. 
We will show inductively that each map $f_{i}: C \cup_{A} B_{i} \rightarrow C \amalg_{A} B_{i}$ is an $(m+n-1)$ equivalence and then conclude that the colimit $C \cup_{A} B \rightarrow C \amalg_{A} B$ is also an $(m+n-1)-$ equivalence. (Here we are using that a sequential colimit of $R$-algebras is the same as the sequential colimit of underlying $R$-modules.)

Note that $f_{0}: C \rightarrow C$ is an isomorphism since $B_{0}=A$. Assume $f_{i-1}$ is a $(m+n-1)-$ equivalence. If $B_{i-1} \rightarrow B_{i}$ is a pushout of a trivial cofibration, then it is a trivial cofibration of $R$-algebras and hence also a trivial cofibration of $R$-modules [18, 4.1(3)]. This uses the fact that $B_{i-1}$ is cofibrant as an $R$-algebra and hence also as an $R$-module. It follows that both $C \cup_{A} B_{i-1} \rightarrow C \cup_{A} B_{i}$ and $C \amalg_{A} B_{i-1} \rightarrow C \amalg_{A} B_{i}$ are weak equivalences. Thus, if $f_{i-1}$ is an $(m+n-1)$-equivalence then so is $f_{i}$.

For the remaining case we have $B_{i}=B_{i-1} \amalg_{T_{R}(K)} T_{R}(L)$ with $K \rightarrow L$ as in Lemma A.3. We show that $f_{i}: C \cup_{A} B_{i} \rightarrow C \amalg_{A} B_{i}$ is the composition of two $(m+n-1)$-equivalences. For the first piece, consider the diagram of pushout squares in $R$-modules:

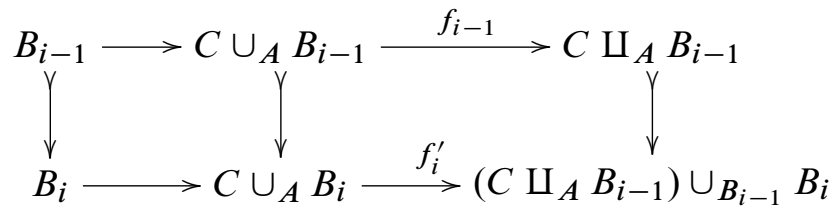

The map $f_{i}^{\prime}$ is an $(m+n-1)$-equivalence since it is the pushout of the $(m+n-1)-$ equivalence $f_{i-1}$. Next consider the pushout of $R$-algebras

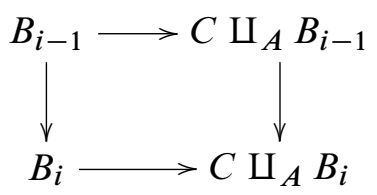

We claim that the top map here is an $(m-1)$-equivalence. Assuming this and recalling that $B_{i}=B_{i-1} \amalg_{T_{R}(K)} T_{R}(L)$, then by Lemma A.5 that $f_{i}^{\prime \prime}:\left(C \amalg_{A} B_{i-1}\right) \cup_{B_{i-1}} B_{i} \rightarrow$ $C \amalg_{A} B_{i}$ is an $(m+n-1)$-equivalence. Since $f_{i}=f_{i}^{\prime \prime} f_{i}^{\prime}$ this would finish the induction step. To establish the claim about the top map above, compare the two horizontal cofibration sequences below.

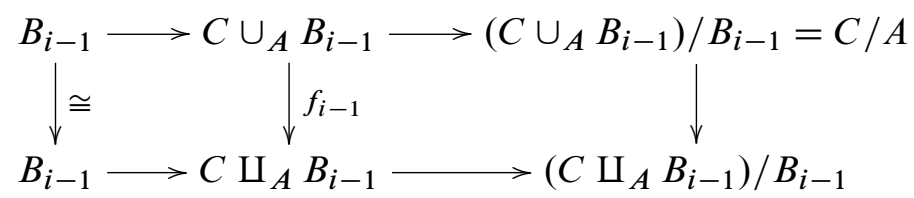

Algebraic 83 Geometric Topology, Volume 6 (2006) 
Since the left vertical map is an isomorphism and the middle map is an $(m+n-1)-$ equivalence the right map is also an $(m+n-1)$-equivalence. Since $C / A$ is $(m-1)-$ connected, so is $\left(C \amalg_{A} B_{i-1}\right) / B_{i-1}$. It follows that $B_{i-1} \rightarrow C \amalg_{A} B_{i-1}$ is an $(m-1)$-equivalence.

\section{References}

[1] M Basterra, André-Quillen cohomology of commutative S-algebras, J. Pure Appl. Algebra 144 (1999) 111-143 MR1732625

[2] M Basterra, M A Mandell, Homology and cohomology of $E_{\infty}$ ring spectra, Math. Z. 249 (2005) 903-944 MR2126222

[3] H-J Baues, Combinatorial foundation of homology and homotopy, Springer Monographs in Mathematics, Springer, Berlin (1999) MR1707308

[4] D Blanc, W G Dwyer, P G Goerss, The realization space of a $\Pi$-algebra: a moduli problem in algebraic topology, Topology 43 (2004) 857-892 MR2061210

[5] D Dugger, Classification spaces of maps in model categories arXiv: math.AT/0604537

[6] D Dugger, Combinatorial model categories have presentations, Adv. Math. 164 (2001) 177-201 MR1870516

[7] D Dugger, B Shipley, Topological equivalences for differential graded algebras arXiv:math. AT/0604259

[8] W G Dwyer, D M Kan, Calculating simplicial localizations, J. Pure Appl. Algebra 18 (1980) 17-35 MR578563

[9] W G Dwyer, D M Kan, Function complexes in homotopical algebra, Topology 19 (1980) 427-440 MR584566

[10] W G Dwyer, D M Kan, A classification theorem for diagrams of simplicial sets, Topology 23 (1984) 139-155 MR744846

[11] P Goerss, M Hopkins, Moduli problems for structured ring spectra, preprint (2005)

[12] M Hovey, Model categories, Mathematical Surveys and Monographs 63, American Mathematical Society, Providence, RI (1999) MR1650134

[13] M Hovey, B Shipley, J Smith, Symmetric spectra, J. Amer. Math. Soc. 13 (2000) 149-208 MR1695653

[14] A Lazarev, Homotopy theory of $A_{\infty}$ ring spectra and applications to $M \mathrm{U}$-modules, $K$-Theory 24 (2001) 243-281 MR1876800

[15] M Mandell, private communication

[16] M A Mandell, B Shipley, A telescope comparison lemma for THH, Topology Appl. 117 (2002) 161-174 MR1875908 
[17] D Quillen, Higher algebraic $K$-theory. $I$, from: "Algebraic $K$-theory, I: Higher $K$ theories (Proc. Conf., Battelle Memorial Inst., Seattle, Wash., 1972)", Springer, Berlin (1973) 85-147. Lecture Notes in Math., Vol. 341 MR0338129

[18] S Schwede, B Shipley, Algebras and modules in monoidal model categories, Proc. London Math. Soc. (3) 80 (2000) 491-511 MR1734325

[19] B Shipley, HZZ-algebra spectra are differential graded algebras, to appear Amer. J. Math. arXiv:math.AT/0209215

Department of Mathematics, University of Oregon

Eugene, OR 97403, USA

Department of Mathematics, University of Illinois at Chicago

Chicago, IL 60607, USA

ddugger@math.uoregon.edu, bshipley@math.uic.edu

Received: 26 July 2006 